\title{
Robust Non-Orthogonal Multiple Access for Aerial and Ground Users
}

\author{
Wee Kiat New, Student Member, IEEE, Chee Yen Leow, Member, IEEE, \\ Keivan Navaie, Senior Member, IEEE, and Zhiguo Ding, Fellow Member, IEEE
}

\begin{abstract}
In this paper, we consider a downlink wireless communication system with the co-existence of ground user (GU) and mobile aerial user (AU). Existing solutions rely on orthogonal multiple access (OMA) to support these users, however, OMA is unable to provide the best rate and outage performance because its spectral efficiency is limited by the users' channel conditions and rate requirements. Thus, we propose an aerialground non-orthogonal multiple access (AG-NOMA) scheme that pairs the GU and $A U$ for data and control links, respectively. Unlike terrestrial non-orthgonal multiple access (NOMA), the key idea of AG-NOMA is to exploit the asymmetric features of the channels and rate demands of the $G U$ and $A U$ in the downlink communication. Based on these opportunities, we investigate the maximum achievable $G U$ rate over a time-varying wireless channel while satisfying the AU Quality-of-Service $(\mathbf{Q o S})$ requirement with perfect and partial channel state information (CSI). For perfect CSI, we derive the optimal successive interference cancellation (SIC) policy, power allocation, GU rate, and feasibility conditions in closed-form expressions. For partial CSI, we also derive the suboptimal SIC policy and power allocation in closed-form expressions, and further discussed a tradeoff between the achievable rate and reliability. This tradeoff depends on the system parameters, and thus we have suggested some appropriate parameters based on theoretical support and standard requirements to strike a balance between rate and reliability. Our simulation results show that AG-NOMA scheme with perfect and partial CSI can achieve up to $+99 \%$ GU rate-improvement as compared to OMA and provide a more sustainable rate-improvement and/or lower outage probability than terrestrial NOMA scheme.
\end{abstract}

Index Terms-Non-Orthogonal Multiple Access, CellularConnected UAV, Optimal SIC policy, Optimal Power Allocation.

\section{INTRODUCTION}

Unmanned aerial vehicle (UAV) has attracted considerable attention in the field of wireless communication. On one hand, it has been used as an aerial communication platform such as aerial base-station and aerial relay. On the other hand, UAV has also gained significant interest in civil applications

This work is jointly supported by H2020-MSCA-RISE-2015 under Grant 690750, the Ministry of Education Malaysia under Grant 07085 and the Universiti Teknologi Malaysia under Grant 19H58 and Grant 04G37. The work of Z. Ding was supported by UK EPSRC under grant number EP/P009719/2. (Corresponding Author: Chee Yen Leow)

Wee Kiat New (email: weekiat@graduate.utm.my) and Chee Yen Leow (email: bruceleow@utm.my) are with the Wireless Communication Centre, School of Electrical Engineering, Faculty of Engineering, Universiti Teknologi Malaysia, 81310 Skudai, Johor, Malaysia; Keivan Navaie (e-mail: k.navaie@lancaster.ac.uk) is with the School of Computing and Communications, Lancaster University, Lancaster, United Kingdom LA1 4WA; Zhiguo Ding (e-mail: zhiguo.ding @manchester.ac.uk) is with the School of Electrical and Electronic Engineering, the University of Manchester, UK. such as agriculture, construction, delivery, and surveillance due to its cost effectiveness, high freedom of mobility, and ease of deployment [1]. To further enhance the potential of these civil applications, the concept of connecting UAV as an aerial user (AU) to the cellular networks, also known as cellular-connected UAV, has been introduced [2], [3]. Existing solutions rely on orthogonal multiple access (OMA) scheme to support ground user (GU) and AU (e.g., [4], [5]). In OMA, the AU and GU are allocated with orthogonal radio resources. Although OMA avoids multi-user interference in a single cell network, it is unable to produce the best rate or outage performance because its spectral efficiency is limited by the users' channel conditions and rate requirements [6], [7].

Meanwhile, the fifth generation (5G) cellular networks and beyond are anticipated to provide higher data rates, lower latency and massive connectivity. To meet these requirements, the non-orthogonal multiple access (NOMA) has been proposed as a key enabling technique [6]-[8]. This scheme relies on the superposition coding (SC) at the transmitter and successive interference cancellation (SIC) at the receiver to share the same radio resource, i.e., the same time/frequency/code. For instance, in a two-user downlink NOMA system, the ground station (GS) would superimpose and transmit the user signals over the same radio resource by allocating more power to the weak user (i.e., user with weaker channel gain) and less power to the strong user (i.e., user with stronger channel gain). On the other end, the weak user would treat the interference as noise to decode its own signal, and the strong user would perform SIC to decode its own signal.

Existing works have investigated the optimal power allocation for terrestrial NOMA systems. For instance, [9]-[11] have presented the optimal power allocation for different types of objectives such as the max-min rate, weighted sum-rate, sum-rate with QoS constraint, weighted energy-efficiency and energy efficiency with QoS constraint in closed-form expressions. Nevertheless, these solutions are limited to the cases where perfect channel state information (CSI) is available. With better understanding over the perfect CSI assumption, researchers are now focusing on optimal power allocation without the availability of perfect CSI [12]-[14]. However, most of the existing works (e.g., [9]-[14]) are limited to the study of instantaneous channel. Besides, they assume there is a specific channel ordering and do not consider the co-existence of GU and AU.

With the presence of $\mathrm{AU}$, one of the key challenges is the interference from the ground nodes. Existing works have analyzed the impact of interference due to the coexistence of GS, 
GU and AU [15]-[18]. These works show that severe downlink interference would occur at the AU when GS is transmitting to a GU due to AU flying or hovering at a higher altitude. Hence, the efficiency of existing interference mitigation techniques such as directional antenna beam selection, power control, inter-cell interference coordination, and coordinated multipoint technique have been evaluated [16], [17].

Unlike [15]-[18], we propose the aerial-ground NOMA (AG-NOMA) to exploit the interference between GU and AU via NOMA. The motivations of pairing the GU and AU over the same radio resource are two folds: asymmetric channels and asymmetric rate demands. Since AU typically experiences a stronger communication link than that of the GU, NOMA may yield a larger rate region due to the distinctive channels [6]. Another reason to pair GU and AU over the same radio resource comes from their rate demand perspective. Given that AU typically has a stronger communication link than that of the GU, yet in the downlink, it is the GU that requires a higher data rate for multimedia applications while the $\mathrm{AU}$ requires a lower data rate but highly reliable communication for its control links [3]. Exploiting these characteristics enables AGNOMA to efficiently foster the asymmetricity between AU and $\mathrm{GU}$, and achieves a higher rate and/or lower outage probability as compared to OMA and terrestrial NOMA.

Note that, pairing $\mathrm{GU}$ and mobile $\mathrm{AU}$ over the same radio resource introduces new challenges even in a single-cell network. For instance, the distance-based ranking (see, [19]) which is used in the existing NOMA to distinguish the strong and weak users is no longer accurate. This is because, given the same horizontal distance, the AU that is hovering at an optimal altitude has the strongest channel gain as compared to the AU hovering at a lower (i.e., shorter distance) or higher altitude (i.e., longer distance) [20]. Due to the 3D mobility of the AU, classifying based on the types of the links (see, [12]) is also not applicable because the elevation angle affects the probability of LOS. More critically, the 3D mobility of the AU changes the large and small-scale fading characteristics (e.g. path loss exponent and fading mean), and thus posing a great challenge to the SIC order and the power allocation. To address these challenges, in this paper, we address the SIC policy and power allocation.

Some studies on NOMA for UAV communication have been carried out but most of the existing works focus on aerial communication platform that serves GU only [21]-[26]. So far, few attentions have been given to UAV as AU. For instance, [27] proposed a cooperative NOMA scheme that exploit the GS backhaul links for SIC operations, [28] proposed a transmission technique that incorporates NOMA and zero-forcing beamforming to mitigate the interference, and [29] proposed an uplink NOMA to serve GU and AU while optimizing the AU trajectory and its cell-association order. Nevertheless, [27]-[29] only focus on the uplink communication. From the AU perspective, some works have investigated the AU trajectory to reduce the outage [30], [31], interference [32] and computational offloading [33]. However, these works focus on OMA. From the system perspective, optimizing the trajectory of the AU is irrelevant in this paper because the AU mobility is controlled by its own operator, not by the network.
Motivated by the above, we consider a downlink wireless communication system with the co-existence of GU and mobile AU. We assume the GS is serving the GU for data links and AU for control links. Since the control links are critical for the safe operation of the UAVs, QoS requirements are imposed. We then investigate the achievable GU rate for data link over a time-varying wireless channel while satisfying the AU QoS requirement for control link by AG-NOMA scheme with perfect and partial CSI. The main contributions of this paper are summarized as follows:

- We formulate an optimization problem that maximizes the achievable GU rate over a time-varying wireless channel by optimal SIC policy and power allocation, subject to the $\mathrm{AU}$ meeting its $\mathrm{QoS}$ requirement with perfect and partial CSI. The solutions to the perfect CSI problem provide the insight for obtaining the solutions for the partial CSI problem.

- For the partial CSI case, we derive the probability of AU/GU channel order in a closed-form expression, where this joint probability involves non-central chi-squared and central chi-squared distributions. This probability enables the recognition of the strong and weak users, thereby allowing the AG-NOMA scheme with partial CSI to achieve a higher GU rate.

- For the partial CSI case, we consider the mobility effect of $\mathrm{AU}$ and obtain the suboptimal power allocation in a closed-form expression. The proposed suboptimal power is dynamically allocated and exploits the complementary cumulative distribution function (CCDF) of the AU channel gain in each time slot to ensure the AU QoS requirement across time.

- In the absence of perfect CSI, there exists a fundamental tradeoff between the achievable GU rate and the reliability of meeting the $\mathrm{AU} \mathrm{QoS}$ requirement. We investigate this tradeoff and further suggest a suboptimal SIC policy and targeted probability based on theoretical support and standard requirements to strike a balance between the achievable rate and the reliability.

Note that unlike terrestrial NOMA, this paper considers the cases where the AU could act as the strong or weak user due to the 3D mobility. In the absence of perfect CSI, the GS may assign the strong user to decode the signal directly and the weak user to perform SIC, which is known as the reverse SIC policy. In contrast to the existing methods (e.g., [12], [19]), our solutions avoid the reverse SIC policy based on probability measure. Due to the co-existence of $\mathrm{AU}$ and $\mathrm{GU}$, a mixture of LOS and NLOS links is also considered. The mixture of LOS and NLOS links is a common environment in the aerial-ground networks [34]. We show that leveraging the asymmetricity characteristics between $\mathrm{AU}$ and $\mathrm{GU}$ further improves the performance as compared to terrestrial NOMA. Furthermore, this work is user-type oriented, i.e., it maximizes the GU rate while satisfying the $\mathrm{AU}$ QoS requirement regardless of whom is being the strong or weak user.

The rest of the paper is organized as follows: Section II presents the system model and details the proposed AGNOMA for downlink communication, Section III studies the 


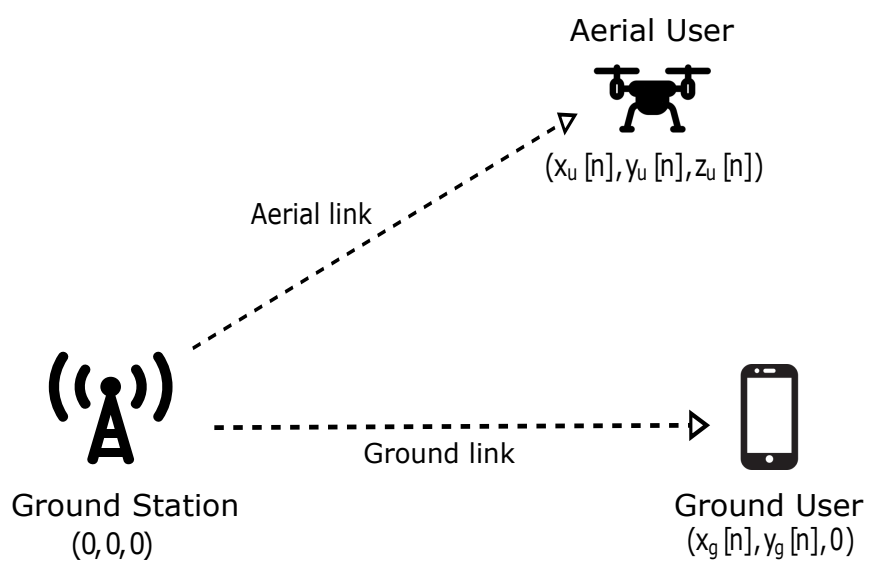

Figure 1: Downlink Communication System with GU and AU.

achievable GU rate over a time-varying wireless channel subject to the AU meeting its QoS requirement by AG-NOMA with perfect CSI. Section IV extends our investigation to the cases where only partial CSI is available and further discusses the tradeoff between the achievable rate and reliability. Then, in Section V, we present our simulation results and finally the findings of this paper are concluded in Section VI.

Notations: In this paper, scalars are denoted by italic letters (e.g., c), and vectors are denoted by boldface letters (e.g., c). $\mathbb{P}(\cdot)$ denotes the probability, $\mathbb{E}[\cdot]$ denotes the statistical expectation, and $\log (\cdot)$ denotes the logarithm with base 2 .

\section{System Model \& The Proposed AG-NOMA SCHEME}

We consider a downlink wireless communication system (see, Fig. 1) with the presence of a $\mathrm{GU}$ and a mobile $\mathrm{AU}$ over $T$ seconds. The duration $T$ is slotted into $N+1$ fixedlength time slots where the interval is larger than the channel coherence time. We assume the GS is located at the origin $(0,0,0)$ throughout the time, the GU is located at the coordinate $\left(x_{g}[n], y_{g}[n], 0\right)$, and the $\mathrm{AU}$ is located at the coordinate $\left(x_{u}[n], y_{u}[n], z_{u}[n]\right)$ at time $n$, where $(x[n], y[n], z[n])$ denotes the 3D-Cartesian coordination of $x$-axis, $y$-axis and z-axis, respectively, with $n=\{1, \ldots, N+1\}$. The distance between the GU and GS at time $n$ is:

$$
d_{g}[n]=\sqrt{x_{g}[n]^{2}+y_{g}[n]^{2}},
$$

and the distance between the AU and GS at time $n$ is:

$$
\hat{d}_{u}[n]=\sqrt{d_{u}[n]^{2}+z_{u}[n]^{2}},
$$

where $d_{u}[n] \triangleq \sqrt{x_{u}[n]^{2}+y_{u}[n]^{2}}$ denotes the horizontal distance between AU and GS at time $n$. Furthermore, this study considers that the communication link between AU and GS follows a probabilistic line-of-sight (LOS)/non line-ofsight (NLOS) model as in [35]-[37]. The probability of LOS between the AU and GS is:

$$
P_{L O S, u}\left(d_{u}[n], z_{u}[n]\right)=\frac{1}{1+a \cdot \exp \{-b \cdot[\phi[n]-a]\}},
$$

where $a$ and $b$ are constant coefficient depending on the environment, and $\phi[n]=\tan ^{-1}\left(\frac{z_{u}[n]}{d_{u}[n]}\right)$ is the elevation angle between the AU and GS at time $n$. The probability of NLOS between the AU and GS is $P_{N L O S, u}\left(d_{u}[n], z_{u}[n]\right)=$ $1-P_{L O S}\left(d_{u}[n], z_{u}[n]\right)$. According to [37], the channel gain between the AU and GS at time $n$ is:

$$
\left|h_{u}[n]\right|^{2}=\frac{A\left|\Omega_{u}[n]\right|^{2}}{\hat{d}_{u}[n]^{\alpha_{u}\left(d_{u}[n], z_{u}[n]\right)}},
$$

where $\alpha_{u}\left(d_{u}[n], z_{u}[n]\right)=\alpha_{e} \cdot P_{N L O S}\left(d_{u}[n], z_{u}[n]\right)+\alpha_{0}$ is the aerial path loss exponent, $\alpha_{0}$ and $\alpha_{e}$ determine the path loss exponent values based on the probability of LOS/NLOS link. In (4), $A$ is a constant coefficient that reflects the effect of operating frequency and antenna gain, and $\left|\Omega_{u}[n]\right|^{2}$ is an i.i.d. random variable that follows a non-central chi-square probability distribution function with 2 degrees of freedom. As shown in [37]-[39], the probability density function of a non-central chi-square with 2 degrees of freedom is:

$$
f_{\Omega}(\omega)=\frac{1}{2 \sigma^{2}} \exp \left\{-\frac{k[n]^{2}+\omega}{2 \sigma^{2}}\right\} I_{0}\left(\frac{k[n]}{\sigma^{2}} \sqrt{\omega}\right) .
$$

In (5), $\omega \geq 0, K[n] \triangleq \frac{k[n]^{2}}{2 \sigma^{2}}$ is the Rician K-factor and $I_{0}(\cdot)$ is the zero-order modified Bessel function of the first kind. According to [37], [38], the Rician K-factor varies according to the elevation angle because larger elevation angle results in higher LOS gain and lower elevation angle results in lower LOS gain. Therefore, the angle-dependent Rician K-factor at time $n$ is:

$$
K[n]=\frac{k_{o}}{2 \sigma^{2}} \cdot \exp \left\{\frac{2}{\pi} \ln \left(\frac{k_{\max }}{k_{o}}\right) \phi[n]\right\},
$$

where $K_{\max } \triangleq \frac{k_{\max }}{2 \sigma^{2}}$ is the maximum Rician K-factor and $K_{o} \triangleq \frac{k_{o}}{2 \sigma^{2}}$ is the minimum Rician K-factor. As observed in (3)-(6), the path loss exponent and Rician $\mathrm{K}$ factor varies according to the $3 \mathrm{D}$ coordinate of $\mathrm{AU}$ at time $n$. However, (3)-(6) are only accurate for high enough altitudes. Therefore, a ground-to-ground link is needed for the GU. According to [37], the channel gain between the GU and GS at time $n$ is:

$$
\left|h_{g}[n]\right|^{2}=\frac{A\left|\Omega_{g}[n]\right|^{2}}{d_{g}[n]},
$$

where $\alpha=\alpha_{e}+\alpha_{0}$ is the ground path loss exponent (NLOS link) and $\left|\Omega_{g}[n]\right|^{2}$ is an i.i.d. random variable that follows a central chi-square probability distribution function with 2 degrees of freedom. As shown in [37], [39], the probability density function of a central chi-square with 2 degrees of freedom is:

$$
f_{\Omega}(\omega)=\frac{1}{2 \sigma^{2}} \exp \left\{-\frac{\omega}{2 \sigma^{2}}\right\} .
$$

In the proposed AG-NOMA scheme, the GU and AU are paired over a radio resource ${ }^{1}$. The pairing consists of two users only to ensure low co-channel interference, hardware complexity, and processing delay. The GS superimposes and

\footnotetext{
${ }^{1}$ Note that, the proposed scheme can be generalized for multiple AUs and GUs in a multi-carrier NOMA system (see, [7]). Specifically, an AU and a GU can be paired over an orthogonal radio resource. Then, each pair of AU and GU can be served by different orthognal radio resource. Sophisticated resource allocation and user pairing among $\mathrm{AU}$ and $\mathrm{GU}$ can improve the performance of multi-carrier AG-NOMA system. This is, however, beyond the scope of this paper.
} 
transmits the desired signals of $\mathrm{AU}$ and $\mathrm{GU}$ with different power coefficients. For ease of expositions, subscript $s$ and $w$ denote the strong and weak users, respectively. According to [6], the transmitted superimposed signal of both $s$ and $w$ users at time $n$ is:

$$
B_{t x}[n]=\sqrt{\rho_{s}[n] P_{t x}} B_{s}[n]+\sqrt{\rho_{w}[n] P_{t x}} B_{w}[n],
$$

where $B_{s}[n]$ and $B_{w}[n]$ are the information bearing at time $n$ for the $s$ and $w$ users, respectively, with $B_{s} \sim C N(0,1)$ and $B_{w} \sim C N(0,1)$. In addition, $\rho_{s}[n]$ and $\rho_{w}[n]$ are the power coefficient for $s$ and $w$ users, respectively, where $\rho_{s}[n]+\rho_{w}[n] \leq 1$ and $P_{t x}$ is the transmission power of GS. The received signal at $s$ user at time $n$ is:

$$
y_{s}[n]=h_{s}[n] B_{t x}[n]+\phi_{s}[n],
$$

where $\phi_{s} \sim C N\left(0, \sigma_{n}^{2}\right)$ is the additive white Gaussian noise. Similarly, the received signal at $w$ user at time $n$ is:

$$
y_{w}[n]=h_{w}[n] B_{t x}[n]+\phi_{w}[n],
$$

where $\phi_{w} \sim C N\left(0, \sigma_{n}^{2}\right)$ is the additive white Gaussian noise. In AG-NOMA, the weak user should decode its signal directly by treating the interference of the strong user as noise while the strong user should perform SIC. As shown in [7], [8], the achievable rate of the $w$ user who decodes the signal directly at time $n$ is:

$$
R_{w}[n]=\log \left(1+\frac{\rho_{w}[n] P_{t x}\left|h_{w}[n]\right|^{2}}{\rho_{s}[n] P_{t x}\left|h_{w}[n]\right|^{2}+\sigma_{n}^{2}}\right),
$$

and the achievable rate of the $s$ user who performs SIC at time $n$ is:

$$
R_{s}[n]=\log \left(1+\frac{\rho_{s}[n] P_{t x}\left|h_{s}[n]\right|^{2}}{\sigma_{n}^{2}}\right) .
$$

According to [7], [8], OMA scheme achieves the following data rate:

$$
R_{i}^{O M A}[n]=\frac{1}{2} \log \left(1+\frac{P_{t x}\left|h_{i}[n]\right|^{2}}{\sigma_{n}^{2}}\right),
$$

where $i \in\{u, g\}$. Here, we assume that the Doppler effect due to the mobility can be perfectly compensated [40]. For partial CSI, this paper utilizes the network localization information and statistical CSI for two reasons. Firstly, the AU/GU 3D coordinate can be obtained up to centimeter accuracy [41], [42], where the AU coordinate must also be known for safety and security purposes [43]. Secondly, the statistical CSI is more stable and can be easily obtained at the GS [44].

\section{AG-NOMA with Perfect Channel State INFORMATION: OPTIMAL TIME-VARYING SIC POLICY AND POWER Allocation}

To obtain the optimal time-varying SIC policy and power allocation, we maximizes the achievable GU rate over a timevarying wireless channel subject to AU meeting its QoS requirement so that the proposed AG-NOMA scheme can reliably support the AU control links and efficiently serve GU data links. With perfect CSI, we formulate the optimization problem as:

$$
\begin{array}{clc}
\max _{\boldsymbol{s}, \boldsymbol{w}, \boldsymbol{\rho}_{\boldsymbol{w}}, \boldsymbol{\rho}_{\boldsymbol{s}},} & \sum_{n=1}^{N+1} R_{g}[n], & \\
\text { s.t. } & R_{u}[n] \geq R_{\text {min }}, & \forall n, \\
& \rho_{w[n]}[n] \geq 0, \rho_{s[n]}[n] \geq 0, & \forall n, \\
& \rho_{w[n]}[n]+\rho_{s[n]}[n] \leq 1, & \forall n, \\
& \rho_{w[n]}[n] \geq \rho_{s[n]}[n], & \forall n, \\
s[n], w[n] \in\{g, u\}, s[n] \neq w[n], & \forall n,
\end{array}
$$

where the objective function (15a) is the achievable GU rate over a time-varying wireless channel as a function of $\left(s, \boldsymbol{w}, \boldsymbol{\rho}_{\boldsymbol{w}}, \boldsymbol{\rho}_{\boldsymbol{s}},\right)$. Constraint (15b) ensures AU meeting its QoS requirement, $(15 \mathrm{c})$ ensures non-negative power coefficients, (15d) ensures feasible power coefficients, (15e) ensures SIC stability in which the power coefficient of the weak user has to be larger than the power coefficient of the strong user in order to decode the weak user signal [11], and (15f) ensures the strong user and weak user are either GU or AU. Unlike similar problems, (15a) and (15b) cannot be evaluated if $s, \boldsymbol{w}$ are unknowns.

Remark 1. Note that, the AU/GU could be strong or weak users and may be assigned in the reverse way. Therefore, in our formulation, (15e) is to ensure the success of SIC operation. According to [11], [45], the power coefficient of the weak user has to be larger than the power coefficient of the strong user to decode the weak user's signal.

In a two-user NOMA scheme, there are two SIC policies: optimal and reverse policies. In optimal policy, the weak user decodes its signal directly and the strong user performs SIC. In the reverse policy, the strong user decodes its signal directly and the weak user performs SIC. Fig. 2 depicts an example of the rate region for optimal and reverse policies, and we present the following lemma.

Lemma 1. There exists a power allocation in the optimal SIC policy that can always outperform the reverse policy. Thus, the optimal time-varying SIC policy for problem (15) at each time $n$ is:

$$
\begin{aligned}
& w^{*}[n]= \begin{cases}u, & \left|h_{u}[n]\right|^{2} \leq\left|h_{g}[n]\right|^{2}, \\
g, & \left|h_{g}[n]\right|^{2}<\left|h_{u}[n]\right|^{2},\end{cases} \\
& s^{*}[n]= \begin{cases}g, & \left|h_{u}[n]\right|^{2} \leq\left|h_{g}[n]\right|^{2}, \\
u, & \left|h_{g}[n]\right|^{2}<\left|h_{u}[n]\right|^{2} .\end{cases}
\end{aligned}
$$

Proof: See Appendix A.

Note that, in contrast to the application of UAV as an aerial base-station or relay, where the UAV movement can be controlled by the network, the GS in this setting has no control over the AU mobility. In particular, the GS only provides downlink communication to the $\mathrm{AU}$, so that control and command $(\mathrm{C} \& \mathrm{C})$ information can be delivered reliably. In practice, it is the AU operator that controls the AU movement. 


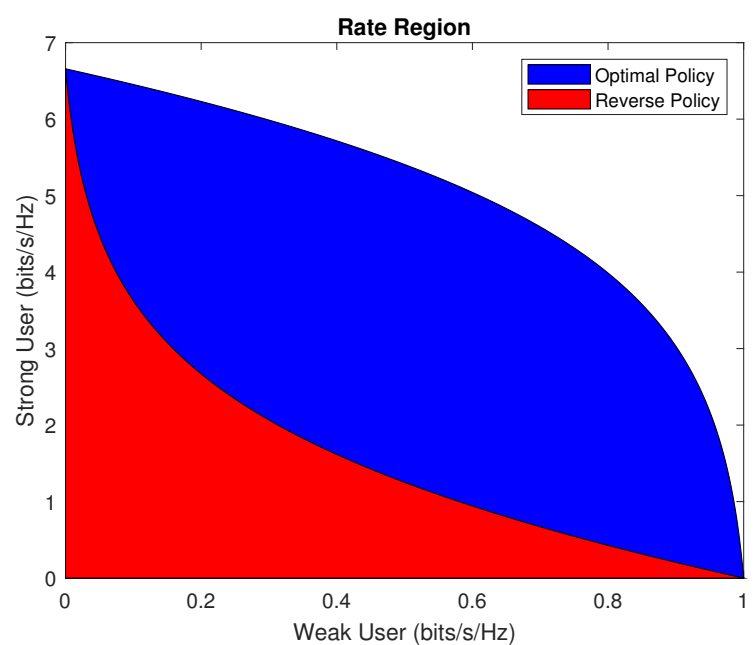

Figure 2: Rate Region of Optimal and Reverse SIC Policy

Hence, (16)-(17) is an important optimal policy from the system perspective as the AU can be a strong or weak user erratically due to its freedom of high mobility in the $3 \mathrm{D}$ space. Given the optimal time-varying SIC policy, (15a)-(15b) are discrete time-varying piecewise functions and (15c)-(15e) change accordingly.

This leads to two cases: (i) AU has a stronger channel gain than that of the GU at time $n$, i.e., $\left|h_{g}[n]\right|^{2}<\left|h_{u}[n]\right|^{2}$, and (ii) $\mathrm{GU}$ has a stronger or equal channel gain than that of the AU at time $n$, i.e., $\left|h_{u}[n]\right|^{2} \leq\left|h_{g}[n]\right|^{2}$. Accordingly, the optimization problem (15) can be decomposed into $N+1$ independent sub-problems. For case (i), the sub-problem is reduced to:

$$
\begin{aligned}
\max _{\rho_{u}[n]} & \log \left(1+\frac{\left(1-\rho_{u}[n]\right) P_{t x}\left|h_{g}[n]\right|^{2}}{\rho_{u}[n] P_{t x}\left|h_{g}[n]\right|^{2}+\sigma_{n}^{2}}\right), \\
\text { s.t. } & \log \left(1+\frac{\rho_{u}[n] P_{t x}\left|h_{u}[n]\right|^{2}}{\sigma_{n}^{2}}\right) \geq R_{\text {min }}, \\
& \frac{1}{2} \geq \rho_{u}[n] \geq 0, \\
& \rho_{g}[n]+\rho_{u}[n]=1 .
\end{aligned}
$$

Proof: See Appendix B.

For case (ii), the sub-problem is reduced to:

$$
\begin{aligned}
\max _{\rho_{u}[n]} & \log \left(1+\frac{\left(1-\rho_{u}[n]\right) P_{t x}\left|h_{g}[n]\right|^{2}}{\sigma_{n}^{2}}\right) \\
\text { s.t. } & \log \left(1+\frac{\rho_{u}[n] P_{t x}\left|h_{u}[n]\right|^{2}}{\left(1-\rho_{u}[n]\right) P_{t x}\left|h_{u}[n]\right|^{2}+\sigma_{n}^{2}}\right) \geq R_{\text {min }} \\
& 1 \geq \rho_{u}[n] \geq \frac{1}{2} \\
& \rho_{u}[n]+\rho_{g}[n]=1 .
\end{aligned}
$$

Proof: See Appendix B.

Lemma 2. The optimal power allocations for sub-problems (18) and (19) is obtained via a one-dimensional searching method or derived in closed-form expressions as follows ${ }^{2}$ :

$$
\rho_{u}^{*}[n]= \begin{cases}\left(2^{R_{\min }}-1\right)\left(\frac{\sigma_{n}^{2}}{P_{t x}\left|h_{u}[n]\right|^{2}}\right), & \left|h_{g}[n]\right|^{2}<\left|h_{u}[n]\right|^{2}, \\ \frac{\left.2^{R_{\min }}-1\right)}{2^{R_{\text {min }}}}\left(1+\frac{\sigma_{n}^{2}}{P_{t x}\left|h_{u}[n]\right|^{2}}\right), & \left|h_{u}[n]\right|^{2} \leq\left|h_{g}[n]\right|^{2},\end{cases}
$$

where, $\rho_{g}^{*}[n]=1-\rho_{u}^{*}[n], \rho_{u}^{*}[n] \in\left[0, \frac{1}{2}\right]$ for $\left|h_{g}[n]\right|^{2}<$ $\left|h_{u}[n]\right|^{2}$, and $\rho_{u}^{*}[n] \in\left[\frac{1}{2}, 1\right]$ for $\left|h_{u}[n]\right|^{2} \leq\left|h_{g}[n]\right|^{2}$.

Proof: To maximize the objective functions in (18) and (19), one has to minimize $\rho_{u}^{*}[n]$ while satisfying (18b) and (19b). Hence, (18b) and (19b) must be satisfied with equality constraint. Rearranging the terms with equality constraint results in (20).

Substituting (20) into the sub-problems, the optimal values for sub-problems (18) and (19) are:

$$
R_{g}^{*}[n]= \begin{cases}\log \left(\frac{\bar{R}_{g}[n]}{\left(2^{R_{m i n}}-1\right) \cdot \xi[n]+1}\right), & \left|h_{g}[n]\right|^{2}<\left|h_{u}[n]\right|^{2}, \\ \log \left(1+\frac{\xi[n] \cdot \bar{R}_{u}[n]}{2^{R_{m i n}}}-\xi[n]\right), & \left|h_{u}[n]\right|^{2} \leq\left|h_{g}[n]\right|^{2},\end{cases}
$$

where $\xi[n]=\frac{\left|h_{g}[n]\right|^{2}}{\left|h_{u}[n]\right|^{2}}, \stackrel{\circ}{R}_{g}[n]=\left(1+\frac{P_{t x}\left|h_{g}[n]\right|^{2}}{2 \sigma_{n}^{2}}\right), \bar{R}_{g}[n]=$ $\left(1+\frac{P_{t x}\left|h_{g}[n]\right|^{2}}{\sigma_{n}^{2}}\right)$, and $\bar{R}_{u}[n]=\left(1+\frac{P_{t x}\left|h_{u}[n]\right|^{2}}{\sigma_{n}^{2}}\right)$. Note that, $R_{g}^{*}[n] \in\left[\log \left(\stackrel{\circ}{R}_{g}[n]\right), \log \left(\bar{R}_{g}[n]\right)\right]$ for $\left|h_{g}[n]\right|^{2}<$ $\left|h_{u}[n]\right|^{2}$ and $R_{g}^{*}[n] \in\left[0, \log \left(\stackrel{\circ}{R}_{g}[n]\right)\right]$ for $\left|h_{u}[n]\right|^{2} \leq$ $\left|h_{g}[n]\right|^{2}$. Furthermore, the feasible conditions at time $n$ is then derived by substituting the feasible power allocation into the constraints (18b-19b). This leads to the following:

$$
\left|h_{u}[n]\right|^{2} \geq \begin{cases}2\left(2^{R_{\min }}-1\right) \frac{\sigma_{n}^{2}}{P_{t x}}, & \left|h_{g}[n]\right|^{2}<\left|h_{u}[n]\right|^{2}, \\ \left(2^{R_{\min }}-1\right) \frac{\sigma_{n}^{2}}{P_{t x}}, & \left|h_{u}[n]\right|^{2} \leq\left|h_{g}[n]\right|^{2} .\end{cases}
$$

Problem (15) is an infeasible problem if one of the $N+1$ sub-problems cannot meet the QoS requirement, however, it is still necessary to handle the system. When the sub-problem is infeasible, we assume $\rho_{u}^{*}[n]=\frac{1}{2}$, if $\left|h_{g}[n]\right|^{2}<\left|h_{u}[n]\right|^{2}$ and $\rho_{u}^{*}[n]=1$, if $\left|h_{u}[n]\right|^{2} \leq\left|h_{g}[n]\right|^{2}$ for safety and SIC stability purposes, i.e., the system tries to serve the AU link with full priority without violating the SIC stability constraint.

\section{AG-NOMA with Partial Channel State}

INFORMATION: SUBOPTIMAL TIME-VARYING SIC POLICY AND POWER Allocation

The above AG-NOMA solution relies on perfect channel state information (CSI). In practice, however, it is difficult to obtain perfect CSI. Thus, we propose AG-NOMA scheme with partial CSI that leverages the network localization information such as user coordinates and the statistical CSI such as the mean and variance. Due to the reliance on statistical information, we refer to this scheme as AG-NOMA scheme

\footnotetext{
${ }^{2}$ In some cases, an arbitrary power difference $\varepsilon>0$ is required between the strong and weak user signals. To cater this requirement, one can rewrite (15e) as $\rho_{w[n]}[n] \geq \rho_{s[n]}[n]+\varepsilon$. Following the same line of argument as presented in this paper, one will obtain the closed-form expressions as presented in (20). However, the domains of $\boldsymbol{\rho}_{s}^{*}$ and $\boldsymbol{\rho}_{\boldsymbol{w}}^{*}$ are respectively changed to $\rho_{s}^{*}[n] \in$ $\left[0, \frac{1-\varepsilon}{2}\right]$ and $\rho_{w}^{*}[n] \in\left[\frac{1+\varepsilon}{2}, 1\right]$ for $\forall n$.
} 
with statistical CSI. The stochastic optimization problem is formulated as:

$$
\begin{array}{ccc}
\max & \sum_{\boldsymbol{s}, \boldsymbol{w}, \boldsymbol{\rho}_{\boldsymbol{w}}, \boldsymbol{\rho}_{\boldsymbol{s}},} \sum_{n=1}^{N+1} R_{g}[n], & \text { (23a) } \\
\text { s.t. } & \mathbb{P}\left(R_{u}[n] \geq R_{\text {min }}\right) \geq \delta_{\text {target }}, & \forall n, \quad \text { (23b) } \\
& \mathbb{P}(s[n]=u, w[n]=g) \geq \delta_{\text {conf }}, & \exists n, \quad(23 \mathrm{c}) \\
& \rho_{w[n]}[n] \geq 0, \rho_{s[n]}[n] \geq 0, & \forall n, \quad(23 \mathrm{~d}) \\
& \rho_{w[n]}[n]+\rho_{s[n]}[n] \leq 1, & \forall n, \quad(23 \mathrm{e}) \\
& \rho_{w[n]}[n] \geq \rho_{s[n]}[n], & \forall n, \quad(23 \mathrm{f}) \\
s[n], w[n] \in\{g, u\}, s[n] \neq w[n], & \forall n, \quad(23 \mathrm{~g})
\end{array}
$$

where the objective function (23a) is the achievable GU rate over a time-varying wireless channel as a function of $\left(s, w, \rho_{w}, \rho_{s}\right)$. Constraint (23b) ensures AU meeting its QoS requirement with a targeted probability, (23c) ensures the strong user is $\mathrm{AU}$ and the weak user is GU with a specific confidence level ${ }^{3}$, (23d) ensures non-negative power coefficients, (23e) ensures feasible power coefficients, (23f) ensures SIC stability, and (23g) ensures the strong user and weak user are either GU or AU. Note that, (23) cannot be solved directly due the absence of perfect CSI. A standard method to solve this problem is to transform (23) into a nonprobabilistic optimization problem.

From Lemma 1, it is seen that to achieve the optimal GU rate performance, it is necessary to correctly distinguish the strong and weak users. Although the perfect CSI is not available, the probability of the AU/GU channel order can be computed based on the statistical CSI.

Lemma 3. The probability of $A U$ as the strong user and $G U$ as the weak user at time $n$ is:

$$
\begin{gathered}
\mathbb{P}\left\{\left|h_{u}[n]\right|^{2}>\left|h_{g}[n]\right|^{2}\right\}=\frac{\sqrt{2} \sigma}{k[n]} \times\left[\exp \left\{\frac{-k[n]^{2}}{4 \sigma^{2}}\right\} \times\right. \\
M_{-\frac{1}{2}, 0}\left(\frac{k[n]^{2}}{2 \sigma^{2}}\right)-\sqrt{\frac{l_{g}[n]}{l_{g}[n]+l_{u}[n]}} \times \\
\left.\exp \left\{-\frac{\left(l_{g}[n]+2 l_{u}[n]\right)}{\left(l_{g}[n]+l_{u}[n]\right)} \frac{k[n]^{2}}{4 \sigma^{2}}\right\} M_{-\frac{1}{2}, 0}\left(\frac{l_{g}[n]}{l_{g}[n]+l_{u}[n]} \cdot \frac{k[n]^{2}}{2 \sigma^{2}}\right)\right]
\end{gathered}
$$

where $M_{\varsigma, \nu}(\cdot)$ is the Whittaker M function.

Proof: See Appendix C.

Lemma 4. The probability of $A U$ as the weak user and $G U$ as the strong user at time $n$ is:

\footnotetext{
${ }^{3}$ As shown in Lemma 1 , the reverse SIC policy should be prevented in general. Therefore, in the absence of perfect CSI, a confidence level is required to ensure the $\mathrm{AU}$ is a strong user and the $\mathrm{GU}$ is a weak user. Note that, $(23 \mathrm{c})$ can be rewritten as $\mathbb{P}(s[n]=g, w[n]=u)>\left(1-\delta_{\text {conf } f}\right)$ for $\exists n$. These constraints are equivalent and complementary across the time. Based on the confidence level, the reverse SIC policy can then be prevented to a certain degree as shown later in this paper.
}

$$
\begin{aligned}
& \mathbb{P}\left\{\left|h_{u}[n]\right|^{2} \leq\left|h_{g}[n]\right|^{2}\right\}=\left(\frac{l_{g}[n]}{l_{g}[n]+l_{u}[n]}\right) \times \\
& \exp \left\{-\left(\frac{l_{u}[n]}{l_{g}[n]+l_{u}[n]}\right) \frac{k[n]^{2}}{2 \sigma^{2}}\right\} .
\end{aligned}
$$

Proof: See Appendix D.

Note that, (24) is a semi-closed form expression because the Whittaker M function involves infinite series. Since we only pair GU and AU, we can derive the probability of AU as the strong user and GU as the weak user in closed-form expression by taking the complementary probability of (25). Now, we can determine the SIC policy with specific confidence level $\delta_{\text {conf }}$ and the suboptimal SIC policy is:

$$
\begin{gathered}
w^{*}[n]= \begin{cases}u, & \mathbb{P}\left\{\left|h_{u}[n]\right|^{2}>\left|h_{g}[n]\right|^{2}\right\}<\delta_{\text {conf }}, \\
g, & \mathbb{P}\left\{\left|h_{u}[n]\right|^{2}>\left|h_{g}[n]\right|^{2}\right\} \geq \delta_{\text {conf }},\end{cases} \\
s^{*}[n]= \begin{cases}g, & \mathbb{P}\left\{\left|h_{u}[n]\right|^{2}>\left|h_{g}[n]\right|^{2}\right\}<\delta_{\text {conf }}, \\
u, & \mathbb{P}\left\{\left|h_{u}[n]\right|^{2}>\left|h_{g}[n]\right|^{2}\right\} \geq \delta_{\text {conf }} .\end{cases}
\end{gathered}
$$

Given the suboptimal SIC policy, the optimization problem (23) is then reformulated as (28), as shown on the top of next page, where, $N_{F}=\left\{n \mid \mathbb{P}\left\{\left|h_{u}[n]\right|^{2}>\left|h_{g}[n]\right|^{2}\right\} \geq \epsilon_{\text {conf } f}\right\}$, and $N_{S}=\left\{n \mid \mathbb{P}\left\{\left|h_{u}[n]\right|^{2}>\left|h_{g}[n]\right|^{2}\right\}<\epsilon_{\text {conf }}\right\}$.

Substituting (4) into (28b)-(28c) and introducing $\omega_{l b}[n]$ as a non-probabilistic variable in replace of random variable $\left|\Omega_{u}[n]\right|^{2}$, we present the following lemma:

Lemma 5. The suboptimal power allocation at time of (28) is:

$$
\rho_{u}^{\star}[n]= \begin{cases}\left(2^{R_{\min }}-1\right) c_{1}[n], & n \in N_{F}, \\ \frac{\left(2^{R_{\min }}-1\right)\left(1+c_{1}[n]\right)}{2^{R_{\text {min }}}}, & n \in N_{s},\end{cases}
$$

where $\rho_{g}^{\star}[n]=1-\rho_{u}^{\star}[n], \quad c_{1}[n]=\frac{\sigma_{n}^{2} \cdot \tilde{d}_{u}[n]^{\alpha_{u}\left(d_{u}[n], z_{u}[n]\right)}}{P_{t x} \cdot A \cdot \omega_{l b}^{*}[n]}$, and $\omega_{l b}^{*}[n]>0$ is the power factor used to counter the uncertainty of small-scale fading effect at time $n$ with a targeted probability, $\delta_{\text {target }}$.

Proof: To maximize the objective function in (28), one has to minimize $\rho_{u}^{*}[n]$ while satisfying (28b) and (28c). Hence, (28b) and (28c) must be satisfied with equality constraint. Let $\omega_{l b}[n]$ be a non-probabilistic variable that can satisfy the AU QoS requirement with a targeted probability. The optimal $\omega_{l b}^{*}[n]$ is obtained via the complementary cumulative distributive function (CCDF) that satisfies the following condition: ${ }^{4}$ :

$$
Q_{1}\left(\sqrt{\frac{\mathbb{E}\left[\left|\Omega_{u}[n]\right|^{2}\right]}{\sigma}}, \sqrt{\frac{\omega_{l b}^{*}[n]}{\sigma}}\right)=\delta_{\text {target }},
$$

where $Q_{1}(\cdot, \cdot)$ is the first order Marcum Q-function. Rearranging the terms with equality constraint results in (29).

Generally, the parameters $\delta_{\text {target }}$ and $\delta_{\text {conf }}$ are system parameters, and these parameters would determine the achievable

\footnotetext{
${ }^{4} \mathrm{Here}$, we exploit the CCDF of the AU channel gain in each time slot to overcome the 3D mobility effect of the AU.
} 


$$
\begin{array}{cc}
\max _{\boldsymbol{u}} & \sum_{n \in N_{F}} \log \left(1+\frac{\left(1-\rho_{u}[n]\right) P_{t x}\left|h_{g}[n]\right|^{2}}{\rho_{u}[n] P_{t x}\left|h_{g}[n]\right|^{2}+\sigma_{n}^{2}}\right)+\sum_{n \in N_{S}} \log \left(1+\frac{\left(1-\rho_{u}[n]\right) P_{t x}\left|h_{g}[n]\right|^{2}}{\sigma_{n}^{2}}\right), \\
\text { s.t. } & \mathbb{P}\left[\log \left(1+\frac{\rho_{u}[n] P_{t x}\left|h_{u}[n]\right|^{2}}{\sigma_{n}^{2}}\right) \geq R_{\text {min }}\right] \geq \delta_{\text {target }}, n \in N_{F}, \\
& \mathbb{P}\left[\log \left(1+\frac{\rho_{u}[n] P_{t x}\left|h_{u}[n]\right|^{2}}{\left(1-\rho_{u}[n]\right) P_{t x}\left|h_{u}[n]\right|^{2}+\sigma_{n}^{2}}\right) \geq R_{\text {min }}\right] \geq \delta_{\text {target }}, n \in N_{S}, \\
\frac{1}{2} \geq \rho_{u}[n] \geq 0, n \in N_{F}, \\
1 \geq \rho_{u}[n] \geq \frac{1}{2}, n \in N_{S}, \\
\rho_{g}[n]+\rho_{u}[n]=1, \forall n .
\end{array}
$$

GU rate, the rate improvement, and the outage probability ${ }^{5}$. Given (29) and (30), it is clear that $\rho_{u}^{\star}$ is an increasing function w.r.t. $\delta_{\text {target }}$. Thus, increasing $\delta_{\text {target }}$ leads to smaller $\rho_{g}^{\star}$, and hence there exists a tradeoff between outage probability and the achievable GU rate. Besides, $\delta_{\text {conf }}$ determines the rate-improvement given by AG-NOMA. If $\delta_{\text {conf }}=1$, the suboptimal SIC policy always assigns $w[n]=u[n]$ and $s[n]=g[n]$ for all $n$ since $\arg \max \mathbb{P}\left\{\left|h_{u}[n]\right|^{2}>\left|h_{g}[n]\right|^{2}\right\}=1$. This approach provides more reliability for the AU to meet its QoS requirement because for $w[n]=u[n], \rho_{u}[n] \in\left[\frac{1}{2}, 1\right]$. However, the achievable GU rate is bounded because for $s[n]=g[n], \rho_{g}[n] \in\left[0, \frac{1}{2}\right]$.

On the contrary, we can achieve a higher GU rate by setting a smaller $\delta_{\text {conf }}$ at the cost of a lower level of reliability. If $\mathbb{P}\left\{\left|h_{u}[n]\right|^{2}>\left|h_{g}[n]\right|^{2}\right\} \geq \delta_{\text {conf }}$, a higher GU rate can be attained for $w[n]=g[n]$ because $\rho_{g}[n] \in\left[\frac{1}{2}, 1\right]$. However, if $\delta_{\text {conf }}$ is too small, the GU rate may be degraded due to higher likelihood where the real order of the channel gain may be the otherwise. In addition, the AU QoS requirement may not be reliably met because if $s[n]=u[n]$, the feasible power allocation is bounded by $\rho_{u}[n] \in\left[0, \frac{1}{2}\right]$. Therefore, it is necessary to choose $\delta_{\text {conf }}$ carefully and thus we present the following lemma:

Lemma 6. The suboptimal SIC policy can assign $s[n]=$ $u, w[n]=g$ if and only if the following inequality holds:

$$
\begin{aligned}
1-\left(2^{R_{\text {min }}}-1\right) c_{1}[n] \geq & \left(\frac{1}{2^{R_{\text {min }}}}+\frac{c_{1}[n]}{2^{R_{\text {min }}}}-c_{1}[n]\right) \times \\
& \left(1+\frac{\left(2^{R_{\text {min }}}-1\right)}{\omega_{l b}} \frac{l_{g}[n]}{l_{u}[n]}\right)
\end{aligned}
$$

Proof: See Appendix E.

According to [17], [46], $\delta_{\text {target }}$ is required to be at least 0.999 for reliable communication. Hence, for AG-NOMA scheme with statistical CSI, it is suggested that the suboptimal SIC policy be assigned according to (31) and $\delta_{\text {target }}=0.999$.

\section{Simulation Results}

We employ Monte-Carlo simulation with 100,000 samples to evaluate the performance of different schemes in supporting

\footnotetext{
${ }^{5}$ The system is said to be in outage at time $n$ when no feasible power allocation can satisfy the AU QoS requirement.
}

GU for the data links and AU for the control links. These include the proposed AG-NOMA scheme with perfect CSI (AGNOMA-PC), the proposed AG-NOMA scheme with statistical CSI (AG-NOMA-SC), existing terrestrial NOMA scheme with perfect CSI, also referred as ground-to-ground NOMA (GGNOMA), and existing OMA scheme.

For AG-NOMA scheme with statistical CSI, the suboptimal SIC policy follows (31) and $\delta_{\text {target }}=0.999$ unless otherwise stated. For all the schemes, we assume there is a "secondary" GU located at a fixed location, $(-100,0,0)$, throughout the time in which the goal is to maximize its rate. On the other end, there is a "primary" AU or "primary" GU being paired with the secondary GU whilst the QoS requirement of the primary user has to be met. The earlier pairing represents AGNOMA and the latter represents GG-NOMA. Note that, we compare different primary users here in order to highlight the benefits and differences introduced by the AU.

The parameters used in the simulation are [36]-[38]: $\alpha_{e}=$ $1.5, \alpha_{0}=2, a=11.95, b=0.136, K_{\max }=15 \mathrm{~dB}, K_{o}=$ $5 \mathrm{~dB}, A=\left(4 \pi f_{c} / c\right)^{-2}, f_{c}=2.0 \mathrm{Ghz}, \sigma_{n}^{2}=-174 \mathrm{dBm}, P_{t x}=$ $-43 \mathrm{dBm}$, and $R_{\min }=1 \mathrm{bits} / \mathrm{s} / \mathrm{Hz}$ unless otherwise stated. For ease of expositions, we directly translate our results from the time domain with $T=24.5 \mathrm{~s} / N=2450$, and $T=23.5 \mathrm{~s} /$ $N=2350$ to the horizontal distance and altitude perspectives (meters), respectively, and the velocity of the UAV is $\nu=$ $20 \mathrm{~m} / \mathrm{s}$ for both cases.

\section{A. Probability of AU/GU Channel Order}

In Fig. 3(a) and Fig. 3(b), the probability of AU/GU channel order over varying horizontal distance and altitude, respectively, are presented. In Fig. 3(a) we assume that the AU is flying at fixed altitude of $120 \mathrm{~m}$ and moving linearly away from the GS and in Fig. 3(b), we assume the AU flying at a fixed horizontal distance of $300 \mathrm{~m}$ and moving vertically upwards. Fig. 3(a) and Fig. 3(b) show that the closed-form expressions respectively given in (24) and (25) are accurate and can be used when the AU is moving in a 3D space.

\section{B. The Achievable Secondary GU Rate}

Fig. 4(a) depicts the achievable secondary GU rate over varying primary user horizontal distance. Here, we assume the AU is flying at a fixed altitude of $120 \mathrm{~m}$ directly above the primary GU while both primary AU and primary GU 


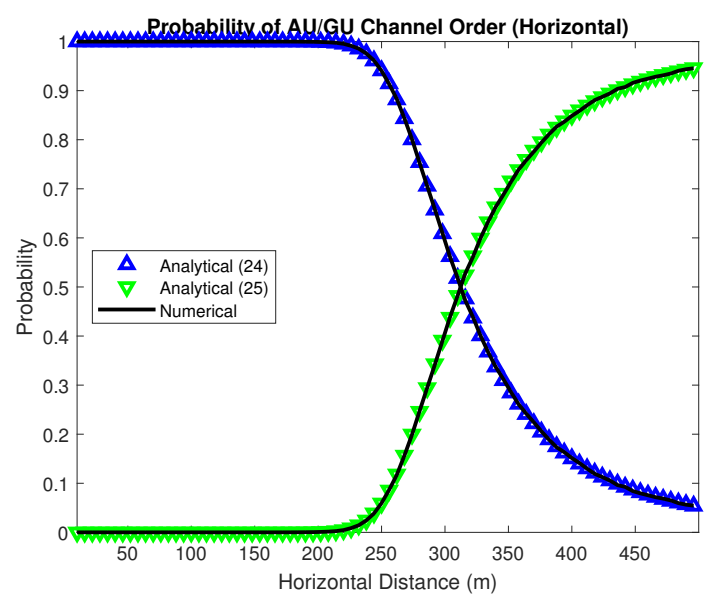

(a)

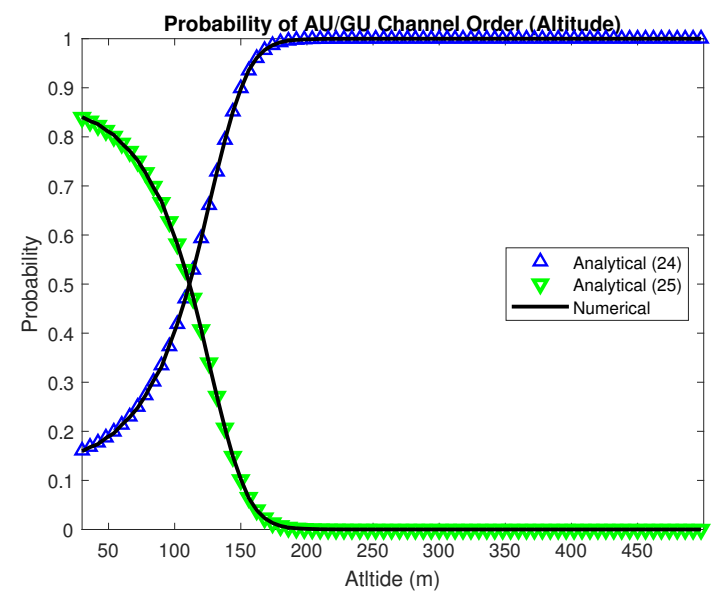

(b)

Figure 3: Probability of AU/GU Channel Order: (a) Horizontal; (b) Altitude.

are moving linearly away from the GS. As seen in the result, all NOMA schemes outperform OMA in terms of the maximum secondary GU rate. Thanks to the spectrum sharing technique, NOMA based scheme can achieve up to $+99 \%$ rate-improvement as compared to OMA. Nevertheless, only AG-NOMA-PC and AG-NOMA-SC can provide a more sustainable rate-improvement than GG-NOMA when the primary user moves horizontally away from the GS.

For instance, AG-NOMA-PC and AG-NOMA-SC can achieve up to $99 \%$ and $98 \%$ rate-improvement, respectively, when the primary AU is $150 \mathrm{~m}$ horizontally away from the GS. The rate-improvement of GG-NOMA is, however, reduced to $67 \%$. As the horizontal distance increases, GG-NOMA may seem to outperform AG-NOMA-SC in terms of the secondary GU rate but in this case GG-NOMA is operating over the unreliable region when the primary user is $60 \mathrm{~m}$ away from the GS. Whereas AG-NOMA-SC is operating over the unreliable region only after $420 \mathrm{~m}$ away. In this paper, the unreliable region implies that numerical outage probability is higher than 0.9985 at that distance or (time $n$ ), whereas reliable region implies the opposite. Overall, the proposed AG-NOMA

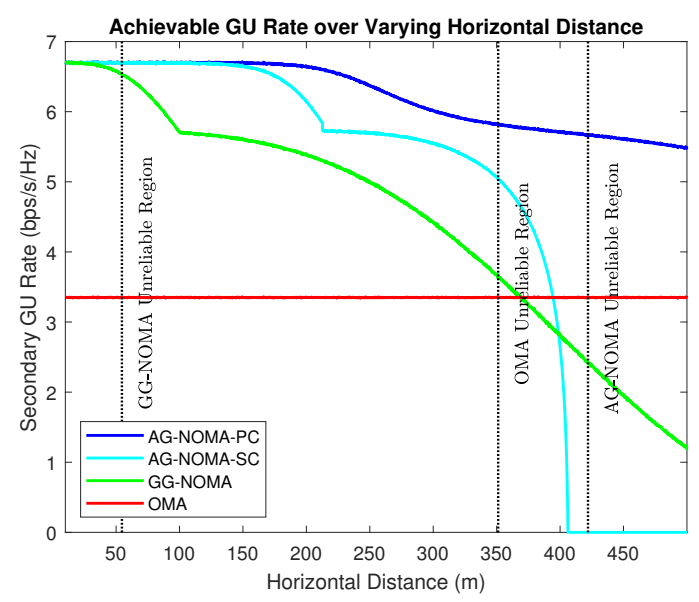

(a)

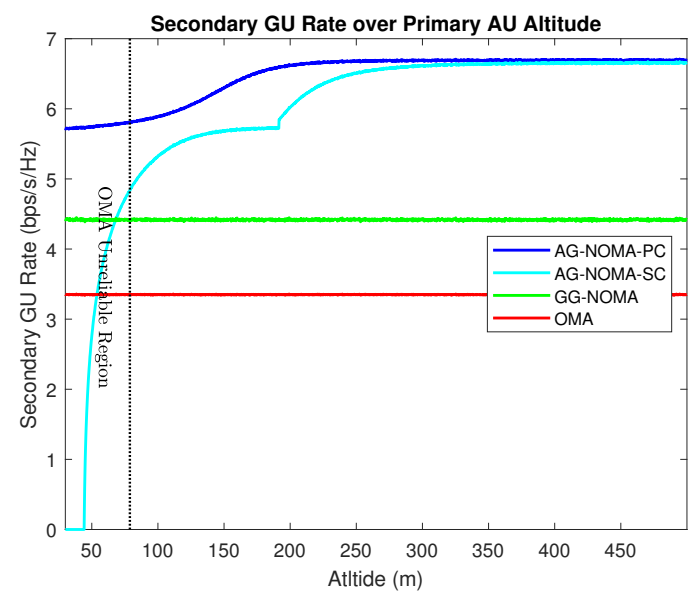

(b)

Figure 4: Achievable Secondary GU Rate: (a) Varying Primary AU/GU Horizontal Distance; (b) Varying Primary AU altitude.

outperforms the GG-NOMA in terms of data rate because the AU experiences dominant LOS link. Therefore, more power can be allocated to the secondary GU to maximize its rate.

It is worthwhile to highlight that there are two bums in Fig. 4(a) on the secondary GU rate of AG-NOMA-PC, AGNOMA-SC and GG-NOMA. This occurs due to the switch of SIC operation from the primary AU/GU to the secondary GU. Specifically, the primary AU/GU will perform the SIC operation when they have stronger channel condition than the secondary GU. As they move further away, the secondary GU will perform the SIC operation whenever the channel gain is stronger than the primary AU/GU.

Fig. 4(b) shows the achievable secondary GU rate over primary user altitude. Here, we assume the primary AU and $\mathrm{GU}$ are located at a fixed horizontal distance of 300m from the GS and only the primary AU flies vertically upwards due to its ability to fly in the air. As observed, AG-NOMAPC, AG-NOMA-SC, and GG-NOMA achieve a maximum rate-improvement of $+99 \%,+98 \%$ and $+33 \%$, respectively. Although GG-NOMA outperforms OMA, GG-NOMA is operating over the unreliable region as the primary GU cannot 
vary its altitude. This is, in fact, one of the advantages of pairing AU-GU over GU-GU, i.e., AU has the ability to fly in the air and establish LOS link with the GS but GU has no such advantage.

Notice that, the GU rate of GG-NOMA is flat because the primary GU is located at a fixed distance and cannot vary its altitude. Meanwhile, the GU rate of OMA scheme is flat because dedicated radio resource is allocated to primary $\mathrm{AU}$ and secondary GU in OMA. Hence, regardless of the AU channel condition or QoS requirement, the secondary GU in OMA scheme only receives half of available radio resource throughout the time.

Although GG-NOMA and OMA outperform AG-NOMA$\mathrm{SC}$ in terms of the achievable secondary $\mathrm{GU}$ rate for some coverage, the numerical outage probability of GG-NOMA is higher than the numerical outage probability of AG-NOMASC. This can be observed in Fig. 5(a) and 5(b). From Fig. 5, it also seen that AG-NOMA-PC has the lowest numerical outage probability due to perfect CSI and spectrum sharing technique while the lowest numerical outage probability of AG-NOMA$\mathrm{SC}$ is bounded to approximately $\left(1-\delta_{\text {target }}\right)$. In general, the proposed AG-NOMA outperforms the GG-NOMA in outage probability because the AU experiences a higher probability of LOS link. Therefore, less power is required to meet the QoS requirement.

\section{Tradeoff Between The Achievable Rate and Reliability}

Fig. 6 investigates the impact of $\delta_{\text {conf }}$ and $\delta_{\text {target }}$ on AGNOMA with statistical CSI to show the trade-off between achievable GU rate and the reliability of meeting the AU QoS requirement. For brevity, we consider AU moving linearly away from the GS with a fixed altitude as for the varying altitude with fixed horizontal distance similar characteristics could be observed. Fig. 6(a) shows the impact of different $\delta_{\text {conf }}$ based on fixed $\delta_{\text {target }}$ and Fig. $6(\mathrm{~b})$ shows the shows the impact of different $\delta_{\text {target }}$.

Fig. 6(a) shows that it is necessary to choose $\delta_{\text {conf }}$ carefully. The appropriate selection of $\delta_{\text {conf }}$ is complex because the GU rate can be degraded when the $\delta_{\text {conf }}$ is too small and the loss of rate-improvement may occur when $\delta_{\text {conf }}$ is too large. Instead of specifying a particular $\delta_{\text {conf }}$, the SIC policy $\hat{\delta}_{\text {target }}$ can be assigned based on (31). As observed in Fig. 6(a), $\hat{\delta}_{\text {target }}+\epsilon$ results in loss of rate-improvement because the switch of SIC operation happens too early. On the other hand, $\hat{\delta}_{\text {target }}-\epsilon$ leads to rate degradation because the switch of SIC operations happens too late. Besides, the rate-improvement in AG-NOMA-SC cannot be fully exploited when $\delta_{\text {conf }}=1$ because the achievable $\mathrm{GU}$ rate is bounded.

Given (31), we can further analyze the impact of $\delta_{\text {target }}$. The targeted probability determines the reliability, where the lowest outage probability would be bounded to $\left(1-\delta_{\text {target }}\right)$ but the achievable secondary GU rate would also be affected by $\delta_{\text {target }}$. As shown in Fig. 6(b), the GU rate would deplete rapidly when $\delta_{\text {target }}$ is large because additional power has to be allocated to the $\mathrm{AU}$ to meet the $\delta_{\text {target }}$ requirement and thus less power is available for the secondary GU rate. This

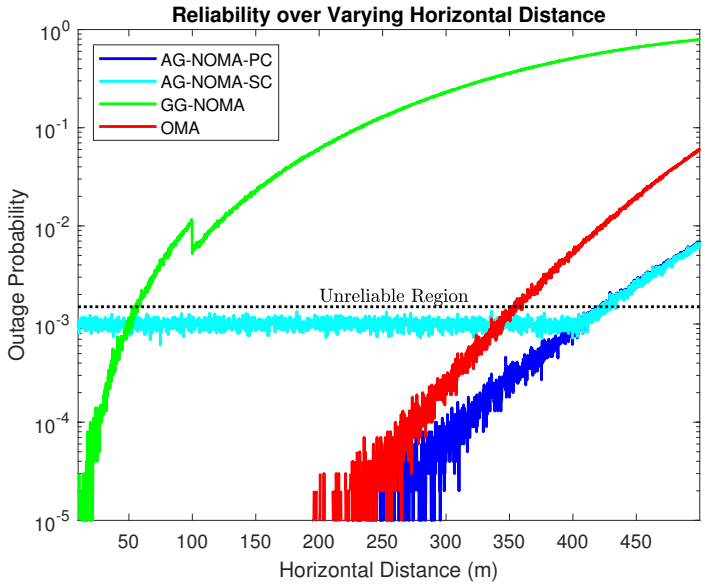

(a)

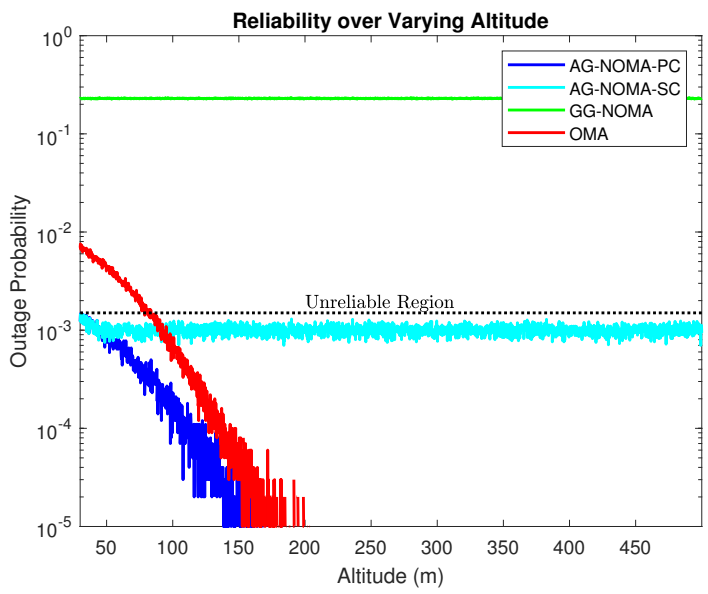

(b)

Figure 5: The Reliability of Meeting QoS Requirement: (a) Varying Primary AU/GU Horizontal Distance; (b) Varying Primary AU Altitude.

impact is also more significant when the GU is a strong user and $\mathrm{AU}$ is weak user. This is anticipated because in the cases where AU is a weak user, all the power can be allocated to AU. On the contrary, when AU is a strong user, at most half of the power can only be allocated to the AU.

\section{CONCLUSION}

In this paper, we have investigated the achievable GU rate over a time-varying wireless channel while satisfying the $\mathrm{AU}$ QoS requirement by AG-NOMA scheme with perfect and statistical CSI. For perfect CSI, we have derived the optimal SIC policy, power allocation, GU rate and feasibility conditions in closed-form expressions. Furthermore, for statistical CSI, we have also derived the suboptimal SIC policy and power allocation in closed-form expressions. The performance of the AG-NOMA scheme with statistical CSI basically depends on the parameters $\delta_{\text {conf }}$ and $\delta_{\text {target }}$ but there exists a tradeoff between the achievable GU rate and reliability of meeting the AU QoS requirement. Specifically, $\delta_{\text {conf }}$ and $\delta_{\text {target }}$ determine the achievable GU rate and outage probability, respectively, 


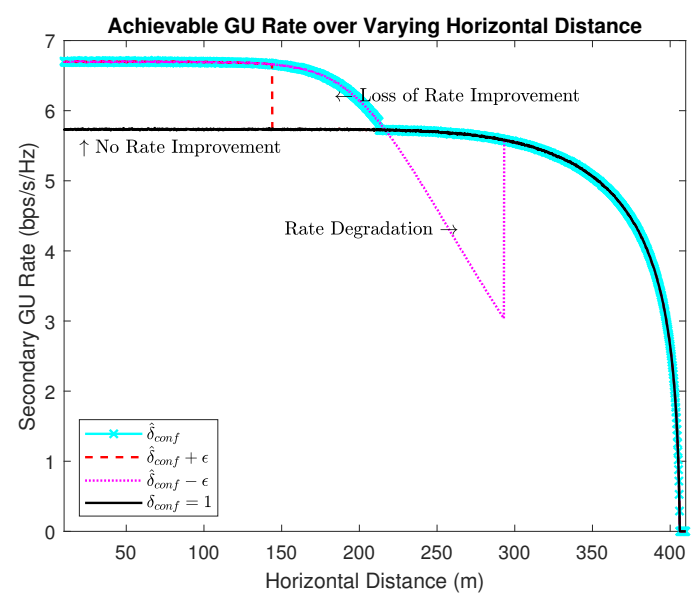

(a)

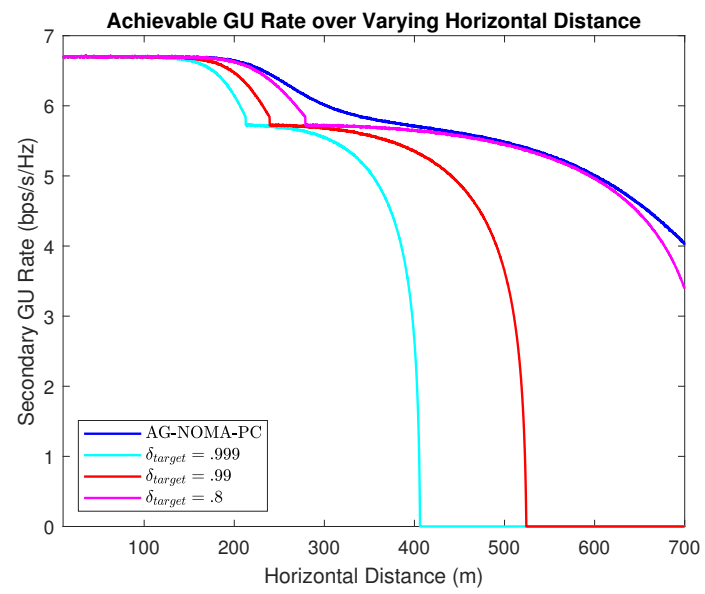

(b)

Figure 6: The Impact of $\delta_{\text {conf }}$ and $\delta_{\text {target }}$ on the achievable GU rate: (a) The Impact of $\delta_{\text {conf }}$ Based on Fixed $\delta_{\text {target }}$; (b) The Impact of Different $\delta_{\text {target }}$.

and thus we suggested appropriate $\delta_{\text {conf }}$ and $\delta_{\text {target }}$ based on theoretical support and requirements to strike a balance between rate and reliability. Our simulation results have indicated that the proposed AG-NOMA scheme with perfect CSI, the proposed AG-NOMA scheme with statistical CSI, and the existing GG-NOMA scheme can potentially achieve up to +99\% GU rate-improvement than OMA scheme. Nevertheless, only the proposed AG-NOMA with perfect and statistical CSI can provide a sustainable rate-improvement over a wider coverage area. With the absence of perfect CSI, AG-NOMA with statistical CSI could also provide better performance in terms of the achievable GU rate and/or outage probability as compared to existing schemes with perfect CSI. This indicates that the proposed AG-NOMA can efficiently serve the GU for data link and reliably support the AU for control link in a single cell network. Nevertheless, AU would suffer a much more severe inter-cell interference than the GU in multi-cell networks due to the LOS link. Therefore, the exploitation of asymmetricity between AU and GU with the presence of intercell interference in multi-cell networks for the proposed AG-
NOMA is still an open problem.

\section{Appendix A: Proof of Lemma 1}

Assume that $\left|h_{s}\right|^{2}>\left|h_{w}\right|^{2}$ for an arbitrary time slot. Following the optimal policy, the achievable rate of the $w$ user that decodes the signal directly is:

$$
R_{w}=\log _{2}\left(1+\frac{\left(1-\rho_{s}\right) P_{t x}\left|h_{w}\right|^{2}}{\rho_{s} P_{t x}\left|h_{w}\right|^{2}+\sigma_{n}^{2}}\right),
$$

and the achievable rate of the $s$ user after performing SIC is:

$$
R_{s}=\log _{2}\left(1+\frac{\rho_{s} P_{t x}\left|h_{s}\right|^{2}}{\sigma_{n}^{2}}\right),
$$

In the reverse policy, the achievable rate of the $w$ user after performing SIC is:

$$
\hat{R}_{w}=\log _{2}\left(1+\frac{\left(1-\hat{\rho}_{s}\right) P_{t x}\left|h_{w}\right|^{2}}{\sigma_{n}^{2}}\right),
$$

and the achievable rate of the $s$ user that decodes the signal directly is:

$$
\hat{R}_{s}=\log _{2}\left(1+\frac{\hat{\rho}_{s} P_{t x}\left|h_{s}\right|^{2}}{\left(1-\hat{\rho}_{s}\right) P_{t x}\left|h_{s}\right|^{2}+\sigma_{n}^{2}}\right) .
$$

From (32)-(35), it is clear that optimal and reverse policies achieve the same achievable rate if $\rho_{s}=\hat{\rho_{s}}=\{0,1\}$. Therefore, we investigate the rate region for optimal and reverse policies for $0<\hat{\rho}_{s}<1$. Let $R_{w}=\hat{R}_{w}$, then rearranging the terms results in:

$$
\left(1-\hat{\rho_{s}}\right)=\frac{\left(1-\rho_{s}\right) \sigma_{n}^{2}}{\rho_{s} P_{t x}\left|h_{s}\right|^{2}+\sigma_{n}^{2}},
$$

and

$$
\hat{\rho}_{s}=\frac{\rho_{s}\left(P_{t x}\left|h_{s}\right|^{2}+\sigma_{n}^{2}\right)}{\rho_{s} P_{t x}\left|h_{s}\right|^{2}+\sigma_{n}^{2}} .
$$

To prove that the optimal policy outperforms the reverse policy, we have to show that $R_{s} \geq \hat{R}_{s}$, which leads to the following inequality:

$$
\rho_{s} \geq \frac{\hat{\rho_{s}}}{\left(1-\hat{\rho_{s}}\right) P_{t x}\left|h_{s}\right|^{2}+\sigma_{n}^{2}} .
$$

Substituting (36) and (37) into (38) results in:

$$
\rho_{s} P_{t x}\left|h_{w}\right|^{2}+\left(1-\rho_{s}\right) P_{t x}\left|h_{s}\right|^{2} \geq P_{t x}\left|h_{w}\right| .
$$

Since $\left|h_{s}\right|^{2}>\left|h_{w}\right|^{2}$, (39) must hold with strict inequality. Hence, the rate region of the reverse policy is the subset of the rate region of the optimal policy. This means there exists a power allocation in the optimal SIC policy that can always outperform the reverse policy. 


\section{APPENDiX B: Proof of Sub-Problem CASE (I) AND (II)}

The proof for case (i) can be obtained following the same line of argument as of case (ii). In fact, it is more straight forward and is thus omitted for brevity. Here, we prove the simplification for case (ii), i.e., $\left|h_{u}[n]\right|^{2} \leq\left|h_{g}[n]\right|^{2}$. Specifically, the subproblem at time $n$ is:

$$
\begin{aligned}
\max _{\rho_{u}[n], \rho_{g[n]}[n]} & \log \left(1+\frac{\rho_{g}[n]\left|h_{g}[n]\right|^{2}}{\sigma_{n}^{2}}\right), \\
\text { s.t. } & \log \left(1+\frac{\rho_{u}[n] P_{t x}\left|h_{u}[n]\right|^{2}}{\rho_{g}[n] P_{t x}\left|h_{u}[n]\right|^{2}+\sigma_{n}^{2}}\right) \geq R_{\text {min }} \\
& \rho_{g}[n] \geq 0, \rho_{u}[n] \geq 0, \\
& \rho_{g}[n]+\rho_{u}[n] \leq 1 \\
& \rho_{g}[n] \geq \rho_{u}[n] .
\end{aligned}
$$

Suppose $R^{\prime}$ is the optimal value of problem (40) with $\rho_{g}^{\prime}[n], \rho_{u}^{\prime}[n]$ as the corresponding feasible optimal solutions, where $\rho_{g}^{\prime}[n]+\rho_{u}^{\prime}[n]<1$. Then, there exist an $\epsilon>0$ such that $\rho_{g}^{\prime}[n]+\rho_{u}^{\prime}[n]+\epsilon=P_{t x}$. One can always increase the the value of (40) by increasing $\rho_{g}^{\prime}[n]$ with some constant $k_{1}$ and $\rho_{u}^{\prime}[n]$ with some constant $k_{2} \geq$ $\left(k_{1} \cdot \rho_{u}[n] P_{t x}\left|h_{u}[n]\right|^{2}\right) /\left(\rho_{g}[n] P_{t x}\left|h_{u}[n]\right|^{2}+\sigma^{2}\right)$, where $k_{1}+k_{2}<\epsilon$. This contradicts that $R^{\prime}$ is the optimal value of (40). Thus, the optimal value of problem (40) must satisfy the condition $\rho_{g}^{*}[n]+\rho_{u}^{*}[n]=1$. Consequently, (40d) can be rewritten as (19d). With this equality constraint, (40c) and (40e) can be rewritten as $(19 \mathrm{c})$.

\section{ApPendix C: Proof of LeMma 3}

For brevity, we drop the time slot index $n$. The probability of $\mathrm{AU}$ to be the strong user and GU to be the weak user is obtained by substituting (4) and (7) as follows:

$$
\mathbb{P}\left\{\left|h_{u}\right|^{2}>\left|h_{g}\right|^{2}\right\}=\mathbb{P}\left\{\frac{A\left|\Omega_{u}\right|^{2}}{\hat{d}_{u}^{\alpha_{u}\left(d_{u}, z_{u}\right)}}>\frac{A\left|\Omega_{g}\right|^{2}}{d_{g}{ }^{\alpha}}\right\} .
$$

Since non-central chi-squared and central chi-squared distributions are closed under scaling by a positive factor, we can rearrange the terms and compute the joint probability as follows:

$$
\begin{aligned}
(41) & =\int_{0}^{\infty} \int_{0}^{\omega_{u}} \frac{1}{l_{g} \cdot 2 \sigma^{2}} \exp \left\{\frac{-\omega_{g}}{l_{g} \cdot 2 \sigma^{2}}\right\} \frac{1}{l_{u} \cdot 2 \sigma^{2}} \times \\
& \exp \left\{\frac{-k^{2}}{2 \sigma^{2}}-\frac{\omega_{u}}{l_{u} \cdot 2 \sigma^{2}}\right\} I_{0}\left(\frac{k}{\sigma^{2}} \cdot \frac{\sqrt{\omega_{u}}}{\sqrt{l_{u}}}\right) d \omega_{g} d \omega_{u},
\end{aligned}
$$

where $l_{g}=\frac{1}{d_{g} \alpha}$ and $l_{u}=\frac{1}{\hat{d}_{u} \alpha_{u}\left(d_{u}, z_{u}\right)}$. Integrating (42) w.r.t. $\omega_{g}$,

$$
\begin{aligned}
(41) & =\frac{1}{l_{u} \cdot 2 \sigma^{2}} \exp \left\{\frac{-k^{2}}{2 \sigma^{2}}\right\}\left[\int_{0}^{\infty} \exp \left\{\frac{-\omega_{u}}{l_{u} \cdot 2 \sigma^{2}}\right\} \times\right. \\
& I_{0}\left(\frac{k}{\sigma^{2}} \cdot \frac{\sqrt{\omega_{u}}}{\sqrt{l_{u}}}\right) d \omega_{u}-\int_{0}^{\infty} \exp \left\{\frac{-\left(l_{u}+l_{g}\right) \omega_{u}}{l_{u} l_{g} 2 \sigma^{2}}\right\} \\
& \left.\times\left(\frac{k}{\sigma^{2}} \cdot \frac{\sqrt{\omega_{u}}}{\sqrt{l_{u}}}\right) d \omega_{u}\right] .
\end{aligned}
$$

According to [47], (pp. 697, $6.614 \quad$ (3)), $\int_{0}^{\infty} \exp \{-\hat{\alpha} x\} I_{2 \nu}(2 \sqrt{\hat{\beta} x}) d x=\frac{\exp \left\{\frac{1}{2} \frac{\hat{\beta}}{\hat{\alpha}}\right\}}{\sqrt{\hat{\alpha} \hat{\beta}}} \cdot \frac{\Gamma(v+1)}{\Gamma(2 \nu+1)} \mathbf{M}_{-\frac{1}{2}, \nu}\left(\frac{\hat{\beta}}{\hat{\alpha}}\right)$, where $\Gamma(\cdot)$ is the gamma function and $\mathbf{M}_{\varsigma, \nu}(\cdot)$ is the Whittaker $\mathrm{M}$ function. Therefore,

$$
\begin{aligned}
(41)= & \frac{\sqrt{2} \sigma}{k}\left[\exp \left\{\frac{-k^{2}}{4 \sigma^{2}}\right\} \mathbf{M}_{-\frac{1}{2}, 0}\left(\frac{k^{2}}{2 \sigma^{2}}\right)-\sqrt{\frac{l_{g}}{l_{g}+l_{u}}} \times\right. \\
& \left.\exp \left\{-\frac{\left(l_{g}+2 l_{u}\right)}{\left(l_{g}+l_{u}\right)} \frac{k^{2}}{4 \sigma^{2}}\right\} \mathbf{M}_{-\frac{1}{2}, 0}\left(\frac{l_{g}}{l_{g}+l_{u}} \cdot \frac{k^{2}}{2 \sigma^{2}}\right)\right] .
\end{aligned}
$$

\section{ApPendix D: Proof of Lemma 4}

For brevity, we drop the time slot index $n$. The probability of $\mathrm{AU}$ to be the weak user and GU to be the strong user is similarly obtained by substituting (4) and (7) as follows:

$$
\mathbb{P}\left\{\left|h_{u}\right|^{2} \leq\left|h_{g}\right|^{2}\right\}=\mathbb{P}\left\{\frac{A\left|\Omega_{u}\right|^{2}}{\hat{d}_{u}^{\alpha_{u}\left(d_{u}, z_{u}\right)}} \leq \frac{A\left|\Omega_{g}\right|^{2}}{d_{g}{ }^{\alpha}}\right\} .
$$

Since non-central chi-squared and central chi-squared distributions are closed under scaling by a positive factor, we can rearrange the terms and compute the joint probability as follows:

$$
\begin{aligned}
(45)= & \int_{0}^{\infty} \int_{0}^{\omega_{g}} \frac{1}{l_{u} \cdot 2 \sigma^{2}} \exp \left\{\frac{-k^{2}}{2 \sigma^{2}}-\frac{\omega_{u}}{l_{u} \cdot 2 \sigma^{2}}\right\} \times \\
& I_{0}\left(\frac{k}{\sigma^{2}} \cdot \frac{\sqrt{\omega_{u}}}{\sqrt{l_{u}}}\right) \frac{1}{l_{g} \cdot 2 \sigma^{2}} \exp \left\{\frac{-\omega_{g}}{l_{g} \cdot 2 \sigma^{2}}\right\} d \omega_{u} d \omega_{g},
\end{aligned}
$$

where $l_{g}=\frac{1}{d_{g} \alpha^{\alpha}}$ and $l_{u}=\frac{1}{\hat{d}_{u}^{\alpha_{u}\left(d_{u}, z_{u}\right)}}$. Integrating (46) w.r.t. $\omega_{u}$, results in

$$
(45)=1-\frac{1}{l_{g} \cdot 2 \sigma^{2}} \int_{0}^{\infty} Q_{1}\left(\frac{k}{\sigma}, \frac{\sqrt{\omega_{g}}}{\sqrt{l_{u} \sigma}}\right) \exp \left\{\frac{-\omega_{g}}{l_{g} \cdot 2 \sigma^{2}}\right\} d \omega_{g} .
$$

In (47), $Q_{1}(\cdot, \cdot)$ is the first order Marcum Qfunction. According to [48], $G(1, \tilde{m}, \tilde{a}, \tilde{b}, \tilde{p})=$ $\int_{0}^{\infty} Q_{\tilde{m}}(\tilde{a}, \tilde{b} \sqrt{\tilde{x}}) \exp \{-\tilde{p} \tilde{x}\} d \tilde{x}$, where

$$
\begin{aligned}
& G(\tilde{k}, \tilde{m}, \tilde{a}, \tilde{b}, \tilde{p})=\frac{\Gamma(\tilde{k})}{\tilde{p}^{\tilde{k}}} \times\left[1-\tilde{a}^{1-\tilde{m}} \exp \left\{-\frac{\tilde{a}^{2}}{2}\right\} \times\right. \\
& \left.\sum_{l=0}^{\tilde{k}-1} \frac{\tilde{p}^{l}}{l ! \tilde{b}^{2 l}} \int_{0}^{\infty} x^{\tilde{m}-2 l} \exp \left\{-\left(\frac{\tilde{p}}{\tilde{b}^{2}}+\frac{1}{2}\right) x\right\} I_{\tilde{m}-1}(\tilde{a} x) d x\right] .
\end{aligned}
$$

Hence, we further simplify (47) as:

$$
\begin{gathered}
(45)=\exp \left\{\frac{-k^{2}}{2 \sigma^{2}}\right\} \int_{0}^{\infty} \omega_{g} \exp \left\{-\left(\frac{l_{g}+l_{u}}{2 \cdot l_{g}}\right) \omega_{g}^{2}\right\} \times \\
I_{0}\left(\frac{k}{\sigma} \cdot \sqrt{\omega_{g}}\right) d \omega_{g}
\end{gathered}
$$

According to [49], (pp. 306, 2.15.5 (4)), $\int_{0}^{\infty} \tilde{x}^{\tilde{\alpha}-1} \exp \left\{-\tilde{p} \tilde{x}^{2}\right\} I_{\tilde{v}}(\tilde{c} x) d x=A_{\tilde{v}}^{\tilde{\alpha}} \quad, \quad$ and $A_{\tilde{v}}^{\tilde{v}+2}=\frac{\tilde{c}^{\tilde{v}}}{(2 \tilde{p})^{\tilde{v}+1}} \exp \left\{\frac{\tilde{c}^{2}}{4 \tilde{p}}\right\}$. Therefore,

$$
(45)=\left(\frac{l_{g}}{l_{g}+l_{u}}\right) \exp \left\{-\left(\frac{l_{u}}{l_{g}+l_{u}}\right) \frac{k^{2}}{2 \sigma^{2}}\right\} \text {. }
$$




\section{Appendix E: PRoOf of Lemma 6}

The achievable $\mathrm{GU}$ rate at time $n$ is $\log \left(1+\frac{\left(1-\rho_{u}^{\star}[n]\right) P_{t x}\left|h_{g}[n]\right|^{2}}{\rho_{u}^{\star}[n] P_{t x}\left|h_{g}[n]\right|^{2}+\sigma_{n}^{2}}\right)$, if $s[n]=u, w[n]=g$, and $\log \left(1+\frac{\left(1-\rho_{u}^{*}[n]\right) P_{t x}\left|h_{g}[n]\right|^{2}}{\sigma_{n}^{2}}\right)$, if $s[n]=g, w[n]=u$. Hence, the optimal SIC policy can assign $s[n]=u, w[n]=g$ in cases where

$$
\frac{\left(1-\rho_{u}^{\star}[n]\right) P_{t x}\left|h_{g}[n]\right|^{2}}{\rho_{u}^{\star}[n] P_{t x}\left|h_{g}[n]\right|^{2}+\sigma_{n}^{2}} \geq \frac{\left(1-\rho_{u}^{\star}[n]\right) P_{t x}\left|h_{g}[n]\right|^{2}}{\sigma_{n}^{2}} .
$$

Since perfect CSI is not available, it is difficult to directly verify (51). However, utilizing the law of large numbers results in:

$$
\mathbb{E}\left[\frac{\left(1-\rho_{u}^{\star}[n]\right) P_{t x}\left|h_{g}[n]\right|^{2}}{\rho_{u}^{\star}[n] P_{t x}\left|h_{g}[n]\right|^{2}+\sigma_{n}^{2}}\right] \geq \mathbb{E}\left[\frac{\left(1-\rho_{u}^{\star}[n]\right) P_{t x}\left|h_{g}[n]\right|^{2}}{\sigma_{n}^{2}}\right] .
$$

Here, the expected value of the left hand side does not exist but the domain of the left hand side is strictly positive. Using Jensen's inequality, we have the following condition:

$$
\frac{\left(1-\rho_{u}^{\star}[n]\right) P_{t x} \mathbb{E}\left|h_{g}[n]\right|^{2}}{\rho_{u}^{\star}[n] P_{t x} \mathbb{E}\left|h_{g}[n]\right|^{2}+\sigma_{n}^{2}} \geq \frac{\left(1-\rho_{u}^{\star}[n]\right) P_{t x} \mathbb{E}\left|h_{g}[n]\right|^{2}}{\sigma_{n}^{2}} .
$$

Substituting the expected value of (7) and (29) into (53), yields:

$$
\begin{aligned}
1-\left(2^{R_{\text {min }}}-1\right) c_{1}[n] \geq & \left(\frac{1}{2^{R_{\text {min }}}}+\frac{c_{1}[n]}{2^{R_{\min }}}-c_{1}[n]\right) \times \\
& \left(1+\frac{\left(2^{R_{\min }}-1\right)}{\omega_{l b}} \frac{l_{g}[n]}{l_{u}[n]}\right),
\end{aligned}
$$

where $c_{1}[n]=\frac{\sigma_{n}^{2} \cdot \tilde{d}_{u}[n]^{\alpha_{u}\left(d_{u}[n], z_{u}[n]\right)}}{P_{t x} \cdot A \cdot \omega_{l b}[n]}$. Hence, the suboptimal SIC policy can assign $s[n]=u, w[n]=g$, if (54) is satisfied.

\section{REFERENCES}

[1] S. Hayat, E. Yanmaz, and R. Muzaffar, "Survey on Unmanned Aerial Vehicle Networks for Civil Applications: A Communications Viewpoint," IEEE Communications Surveys Tutorials, vol. 18, no. 4, pp. 2624-2661, Fourthquarter 2016.

[2] A. Fotouhi, H. Qiang, M. Ding, M. Hassan, L. G. Giordano, A. GarciaRodriguez, and J. Yuan, "Survey on UAV Cellular Communications: Practical Aspects, Standardization Advancements, Regulation, and Security Challenges," IEEE Communications Surveys Tutorials, pp. 1-1, 2019.

[3] Y. Zeng, J. Lyu, and R. Zhang, "Cellular-Connected UAV: Potential, Challenges, and Promising Technologies," IEEE Wireless Communications, vol. 26, no. 1, pp. 120-127, February 2019.

[4] F. Song, J. Li, M. Ding, L. Shi, F. Shu, M. Tao, W. Chen, and H. V. Poor, "Probabilistic Caching for Small-Cell Networks With Terrestrial and Aerial Users," IEEE Transactions on Vehicular Technology, vol. 68, no. 9, pp. 9162-9177, Sep. 2019.

[5] M. M. Azari, F. Rosas, A. Chiumento, and S. Pollin, "Coexistence of Terrestrial and Aerial Users in Cellular Networks," in 2017 IEEE Globecom Workshops (GC Wkshps), Dec 2017, pp. 1-6.

[6] L. Dai, B. Wang, Z. Ding, Z. Wang, S. Chen, and L. Hanzo, "A Survey of Non-Orthogonal Multiple Access for 5G," IEEE Communications Surveys Tutorials, vol. 20, no. 3, pp. 2294-2323, thirdquarter 2018.

[7] Z. Ding, X. Lei, G. K. Karagiannidis, R. Schober, J. Yuan, and V. K Bhargava, "A Survey on Non-Orthogonal Multiple Access for 5G Networks: Research Challenges and Future Trends," IEEE Journal on Selected Areas in Communications, vol. 35, no. 10, pp. 2181-2195, Oct 2017.
[8] Z. Ding, P. Fan, and H. V. Poor, "Impact of User Pairing on 5G Nonorthogonal Multiple-Access Downlink Transmissions," IEEE Transactions on Vehicular Technology, vol. 65, no. 8, pp. 6010-6023, Aug 2016.

[9] Z. Yang, Z. Ding, P. Fan, and N. Al-Dhahir, "A General Power Allocation Scheme to Guarantee Quality of Service in Downlink and Uplink NOMA Systems," IEEE Transactions on Wireless Communications, vol. 15 , no. 11 , pp. 7244-7257, Nov 2016.

[10] Z. Chen, Z. Ding, X. Dai, and R. Zhang, "An Optimization Perspective of the Superiority of NOMA Compared to Conventional OMA," IEEE Transactions on Signal Processing, vol. 65, no. 19, pp. 5191-5202, Oct 2017.

[11] J. Zhu, J. Wang, Y. Huang, S. He, X. You, and L. Yang, "On Optimal Power Allocation for Downlink Non-Orthogonal Multiple Access Systems," IEEE Journal on Selected Areas in Communications, vol. 35, no. 12, pp. 2744-2757, Dec 2017.

[12] S. Guo and X. Zhou, "Robust Resource Allocation With Imperfect Channel Estimation in NOMA-Based Heterogeneous Vehicular Networks," IEEE Transactions on Communications, vol. 67, no. 3, pp. 2321-2332, March 2019.

[13] M. R. Zamani, M. Eslami, M. Khorramizadeh, and Z. Ding, "EnergyEfficient Power Allocation for NOMA With Imperfect CSI," IEEE Transactions on Vehicular Technology, vol. 68, no. 1, pp. 1009-1013, Jan 2019.

[14] F. Fang, H. Zhang, J. Cheng, S. Roy, and V. C. M. Leung, "Joint User Scheduling and Power Allocation Optimization for Energy-Efficient NOMA Systems With Imperfect CSI," IEEE Journal on Selected Areas in Communications, vol. 35, no. 12, pp. 2874-2885, Dec 2017.

[15] X. Lin, V. Yajnanarayana, S. D. Muruganathan, S. Gao, H. Asplund, H. Maattanen, M. Bergstrom, S. Euler, and Y. E. Wang, "The Sky Is Not the Limit: LTE for Unmanned Aerial Vehicles," IEEE Communications Magazine, vol. 56, no. 4, pp. 204-210, APRIL 2018.

[16] I. Kovacs, R. Amorim, H. C. Nguyen, J. Wigard, and P. Mogensen, "Interference Analysis for UAV Connectivity over LTE Using Aerial Radio Measurements," in 2017 IEEE 86th Vehicular Technology Conference (VTC-Fall), Sept 2017, pp. 1-6.

[17] H. C. Nguyen and R. Amorim and J. Wigard and I. Z. Kovács and T. B. Sørensen and P. E. Mogensen, "How to Ensure Reliable Connectivity for Aerial Vehicles Over Cellular Networks," IEEE Access, vol. 6, pp. $12304-12317,2018$

[18] J. Stanczak, I. Z. Kovacs, D. Koziol, J. Wigard, R. Amorim, and H. Nguyen, "Mobility Challenges for Unmanned Aerial Vehicles Connected to Cellular LTE Networks," in 2018 IEEE 87th Vehicular Technology Conference (VTC Spring), June 2018, pp. 1-5.

[19] M. Salehi, H. Tabassum, and E. Hossain, "Accuracy of DistanceBased Ranking of Users in the Analysis of NOMA Systems," IEEE Transactions on Communications, vol. 67, no. 7, pp. 5069-5083, July 2019.

[20] W. Khawaja, I. Guvenc, D. W. Matolak, U. Fiebig, and N. Schneckenberger, "A Survey of Air-to-Ground Propagation Channel Modeling for Unmanned Aerial Vehicles," IEEE Communications Surveys Tutorials, pp. $1-1,2019$

[21] P. K. Sharma and D. I. Kim, "UAV-Enabled Downlink Wireless System with Non-Orthogonal Multiple Access," in 2017 IEEE Globecom Workshops (GC Wkshps), Dec 2017, pp. 1-6.

[22] M. F. Sohail, C. Y. Leow, and S. Won, "Non-Orthogonal Multiple Access for Unmanned Aerial Vehicle Assisted Communication," IEEE Access, vol. 6, pp. 22 716-22 727, 2018.

[23] N. Rupasinghe, Y. Yapici, I. Guvenc, and Y. Kakishima, "NonOrthogonal Multiple Access for mmWave Drone Networks With Limited Feedback," IEEE Transactions on Communications, vol. 67, no. 1, pp. 762-777, Jan 2019.

[24] Q. Wu, J. Xu, and R. Zhang, "Capacity Characterization of UAVEnabled Two-User Broadcast Channel," IEEE Journal on Selected Areas in Communications, pp. 1-1, 2018.

[25] T. Hou, Y. Liu, Z. Song, X. Sun, and Y. Chen, "Exploiting NOMA for UAV Communications in Large-Scale Cellular Networks," IEEE Transactions on Communications, vol. 67, no. 10, pp. 6897-6911, Oct 2019.

[26] Y. Liu, Z. Qin, Y. Cai, Y. Gao, G. Y. Li, and A. Nallanathan, "UAV Communications Based on Non-Orthogonal Multiple Access," IEEE Wireless Communications, vol. 26, no. 1, pp. 52-57, February 2019.

[27] W. Mei and R. Zhang, "Uplink Cooperative NOMA for CellularConnected UAV," IEEE Journal of Selected Topics in Signal Processing, pp. 1-1, 2019.

[28] L. Liu, S. Zhang, and R. Zhang, "Exploiting NOMA for Multi-Beam UAV Communication in Cellular Uplink," in ICC 2019 - 2019 IEEE 
International Conference on Communications (ICC), May 2019, pp. $1-$ 6.

[29] X. Mu, Y. Liu, L. Guo, and J. Lin, "Non-Orthogonal Multiple Access for Air-to-Ground Communication," CoRR, vol. abs/1906.06523, 2019.

[30] S. Zhang and R. Zhang, "Trajectory Design for Cellular-Connected UAV Under Outage Duration Constraint," in ICC 2019 - 2019 IEEE International Conference on Communications (ICC), May 2019, pp. 16.

[31] E. Bulut and I. Guevenc, "Trajectory Optimization for CellularConnected UAVs with Disconnectivity Constraint," in 2018 IEEE International Conference on Communications Workshops (ICC Workshops), May 2018, pp. 1-6.

[32] U. Challita, W. Saad, and C. Bettstetter, "Interference Management for Cellular-Connected UAVs: A Deep Reinforcement Learning Approach," IEEE Transactions on Wireless Communications, vol. 18, no. 4, pp. 2125-2140, April 2019.

[33] X. Cao, J. Xu, and R. Zhang, "Mobile Edge Computing for CellularConnected UAV: Computation Offloading and Trajectory Optimization," in 2018 IEEE 19th International Workshop on Signal Processing Advances in Wireless Communications (SPAWC), June 2018, pp. 1-5.

[34] J. Lyu and R. Zhang, "Network-Connected UAV: 3-D System Modeling and Coverage Performance Analysis," IEEE Internet of Things Journal, vol. 6, no. 4, pp. 7048-7060, Aug 2019.

[35] A. Al-Hourani, S. Kandeepan, and S. Lardner, "Optimal LAP Altitude for Maximum Coverage," IEEE Wireless Communications Letters, vol. 3 , no. 6, pp. 569-572, Dec 2014.

[36] M. Mozaffari, W. Saad, M. Bennis, and M. Debbah, "Mobile Unmanned Aerial Vehicles (UAVs) for Energy-Efficient Internet of Things Communications," IEEE Transactions on Wireless Communications, vol. 16 , no. 11, pp. 7574-7589, Nov 2017.

[37] M. M. Azari, F. Rosas, K. C. Chen, and S. Pollin, "Ultra Reliable UAV Communication Using Altitude and Cooperation Diversity," IEEE Transactions on Communications, vol. 66, no. 1, pp. 330-344, Jan 2018.

[38] X. Yuan, Z. Feng, W. Xu, W. Ni, J. A. Zhang, Z. Wei, and R. P. Liu, "Capacity Analysis of UAV Communications: Cases of Random Trajectories," IEEE Transactions on Vehicular Technology, vol. 67, no. 8, pp. 7564-7576, Aug 2018.

[39] B. B. Haile et al., "Co-channel Interference in Heterogeneous Networks: Rician/Rayleigh Scenario," Master Thesis, Department of Communications and Networking, AALTO UNIVERSITY, 2010.

[40] Y. Zeng and R. Zhang, "Energy-Efficient UAV Communication With Trajectory Optimization," IEEE Transactions on Wireless Communications, vol. 16, no. 6, pp. 3747-3760, June 2017.

[41] C. Laoudias, A. Moreira, S. Kim, S. Lee, L. Wirola, and C. Fischione, "A Survey of Enabling Technologies for Network Localization, Tracking, and Navigation," IEEE Communications Surveys Tutorials, vol. 20, no. 4, pp. 3607-3644, Fourthquarter 2018.

[42] W. Hedgecock, M. Maroti, J. Sallai, P. Volgyesi, and A. Ledeczi, "High-Accuracy Differential Tracking of Low-cost GPS Receivers," in Proceeding of the 11th Annual International Conference on Mobile Systems, Applications, and Services, ser. MobiSys '13. New York, NY, USA: ACM, 2013, pp. 221-234.

[43] G. Ding, Q. Wu, L. Zhang, Y. Lin, T. A. Tsiftsis, and Y. Yao, "An Amateur Drone Surveillance System Based on the Cognitive Internet of Things," IEEE Communications Magazine, vol. 56, no. 1, pp. 29-35, Jan 2018.

[44] S. Qiu, , D. Qu, K. Luo, and T. Jiang, "Downlink Precoding With Mixed Statistical and Imperfect Instantaneous CSI for Massive MIMO Systems," IEEE Transactions on Vehicular Technology, vol. 67, no. 4, pp. 3028-3041, April 2018.

[45] M. S. Ali, H. Tabassum, and E. Hossain, "Dynamic User Clustering and Power Allocation for Uplink and Downlink Non-Orthogonal Multiple Access (NOMA) Systems," IEEE Access, vol. 4, pp. 6325-6343, 2016.

[46] I. Z. Kovács, J. Wigard, P. Mogensen, R. Amorim, T. Sørensen, and S. Hansen, "Enabling BVLOS flights through Cellular Connectivity," 2018, NOKIA Bell Labs.

[47] I. Gradshteyn and I. Ryzhik, Table of Integrals, Series, and Products, 7th edition, A. Jeffrey and D. Zwillinger, Eds. Elsevier Academic Press, Feb 2007.

[48] P. C. Sofotasios, S. Muhaidat, G. K. Karagiannidis, and B. S. Sharif, "Solutions to Integrals Involving the Marcum $Q$-Function and Applications," IEEE Signal Processing Letters, vol. 22, no. 10, pp. 1752-1756, Oct 2015.

[49] Y. Byshkov and O. Marichev, "Integrals and Series. Special Functions," 1983.

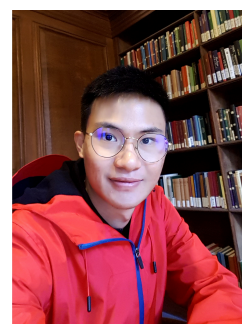

Wee Kiat New received his B.IT in Data Communications and Networking from Multimedia University and M.Eng.Sc from University Malaya in 2012 and 2015, respectively. He is currently pursuing his $\mathrm{Ph} . \mathrm{D}$ in Electrical Engineering at Wireless Communication Centre, Universiti Teknologi Malaysia. He was a visiting researcher at School of Computing and Communications, Lancaster University, and KIOS Research and Innovation Centre, University of Cyprus. His current research interests are optimization, stochastic processes, non-orthogonal multiple access (NOMA), and unmanned aerial Vehicle (UAV) communication.

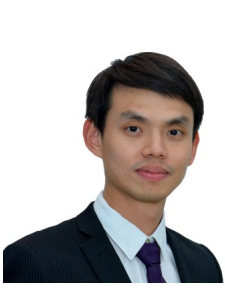

Chee Yen (Bruce) Leow obtained the B.Eng. degree in Computer Engineering from Universiti Teknologi Malaysia (UTM) and the Ph.D. degree from Imperial College London in 2007 and 2011, respectively. Since July 2007, he has been an academic staff with the School of Electrical Engineering, Faculty of Engineering, UTM. He is currently an Associate Professor in the School and a Research Fellow in the Wireless Communication Centre UTM and UTMEricsson Innovation Centre for 5G. His research interests include non-orthogonal multiple access, UAV communication, MIMO, hybrid beamforming, physical layer security, wireless power transfer and prototype development using software defined radio, for $5 \mathrm{G}$ and IoT applications.

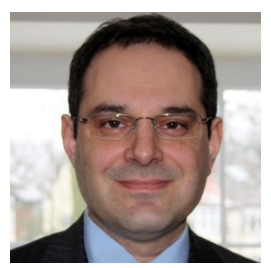

Keivan Navaie is with the School of Computing and Communications, Lancaster University, UK. His research seeks innovative inter-disciplinary solutions to address fundamental technical and design challenges in Intelligent Cyber Physical Systems and their integrations into day-to-day life to improve its sustainability and anti-fragility. Keivan's research covers topics across the following disciplines: Distributed Cloud and Edge Computing, Connectivity Resilience in Cyber-Physical Systems and IoT, Applications of Machine Learning and Artificial Intelligence, and Cognitive Communications Networks.

Keivan is on the editorial board of the IEEE Comm. Letters, IEEE Comm. Surveys and Tutorials, Trans. on Wireless Comm., and IEEE Access. He is also an advocate of evidence-based policy making through his involvement with the EU Environment Agency, and EU Parliament as an Independent Scientific Expert. Dr. Navaie is a Senior Member of the IEEE, and a Chartered Engineer in the UK.

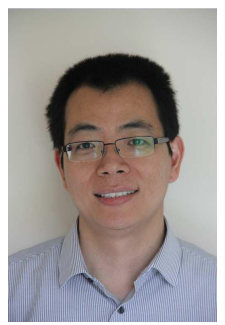

Zhiguo Ding (S'03-M'05-F'20) received his B.Eng in Electrical Engineering from the Beijing University of Posts and Telecommunications in 2000, and the $\mathrm{Ph} . \mathrm{D}$ degree in Electrical Engineering from Imperial College London in 2005. From Jul. 2005 to Apr. 2018, he was working in Queen's University Belfast, Imperial College, Newcastle University and Lancaster University. Since Apr. 2018, he has been with the University of Manchester as a Professor in Communications. From Oct. 2012 to Sept. 2020, he has also been an academic visitor in Princeton University. Dr Ding' research interests are 5G networks, game theory, cooperative and energy harvesting networks and statistical signal processing.

$\mathrm{He}$ is serving as an Area Editor for the IEEE Open Journal of the Communications Society, an Editor for IEEE Transactions on Communications, IEEE Transactions on Vehicular Technology, and Journal of Wireless Communications and Mobile Computing, and was an Editor for IEEE Wireless Communication Letters, IEEE Communication Letters from 2013 to 2016. He received the best paper award in IET ICWMC-2009 and IEEE WCSP-2014, the EU Marie Curie Fellowship 2012-2014, the Top IEEE TVT Editor 2017, IEEE Heinrich Hertz Award 2018, IEEE Jack Neubauer Memorial Award 2018, IEEE Best Signal Processing Letter Award 2018, and Web of Science Highly Cited Researcher 2019. 